\title{
Continuous interior penalty finite element method for the time-dependent Navier-Stokes equations: space discretization and convergence
}

\author{
Erik Burman • Miguel A. Fernández
}

Received: 13 July 2005 / Revised: 13 December 2006 / Published online: 16 May 2007

(C) Springer-Verlag 2007

\begin{abstract}
This paper focuses on the numerical analysis of a finite element method with stabilization for the unsteady incompressible Navier-Stokes equations. Incompressibility and convective effects are both stabilized adding an interior penalty term giving $L^{2}$-control of the jump of the gradient of the approximate solution over the internal faces. Using continuous equal-order finite elements for both velocities and pressures, in a space semi-discretized formulation, we prove convergence of the approximate solution. The error estimates hold irrespective of the Reynolds number, and hence also for the incompressible Euler equations, provided the exact solution is smooth.
\end{abstract}

Mathematics Subject Classification (2000) $35 \mathrm{Q} 30 \cdot 76 \mathrm{D} 05 \cdot 76 \mathrm{M} 10 \cdot 65 \mathrm{~N} 30$

\section{Contents}

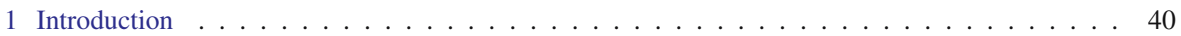

2 The time-dependent Navier-Stokes equations . . . . . . . . . . . . . . . . . . . . 41

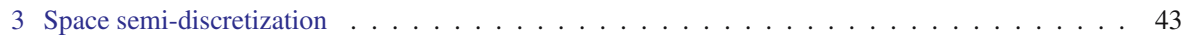

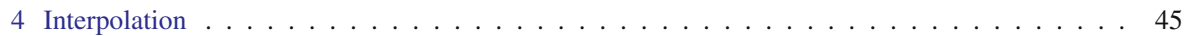

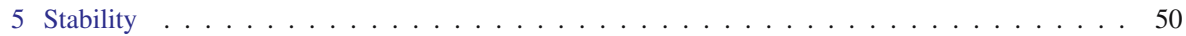

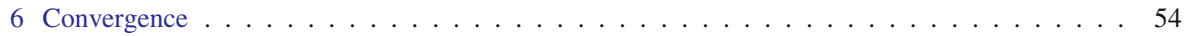

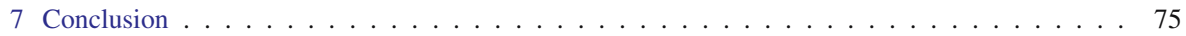

E. Burman $(\varangle)$

Ecole Polytechnique Fédérale de Lausanne, Institut d'analyse et de calcul scientifique,

Station 8, CH-1015 Lausanne, Switzerland

e-mail: erik.burman@epfl.ch

M. A. Fernández

INRIA, REO team, Rocquencourt BP 105, 78153 Le Chesnay Cedex, France 


\section{Introduction}

In this paper we propose a finite element method using interior penalty stabilization for the incompressible Navier-Stokes equations. This method was introduced by Burman and Hansbo in [12], as an extension of the interior penalty method proposed by Douglas and Dupont in [17] to the case of pure transport problems or convection-dominated problems. Pressure stabilization for the Stokes problem was then considered by Burman and Hansbo in [11] and the Oseen's problem was analyzed by Burman et al. in [10]. In the latter, a priori error estimates that hold uniformly in the Reynolds number were proven for sufficiently smooth solutions. In this paper we focus on the time dependent, non-linear Navier-Stokes equations. There exists a vast literature on finite element methods for the Navier-Stokes equations. Let us cite the monograph of Girault and Raviart [20] and the series of papers by Heywood and Rannacher [25-27]. In the case of stabilized finite elements using SUPG-like stabilizations, we cite the work of Johnson and Saranen [30] on a velocity-vorticity formulation, and the paper by Hansbo and Szepessy on the velocity-pressure formulation [23]. Other relevant works on the Navier-Stokes equations include the paper by Tobiska and Verführt [35], the work by Blasco and Codina [15], the work on stabilized mixed methods for the Navier-Stokes equations by He, Lin and Sun [24], and the work on numerical methods for LES using hyperviscosity by Guermond and Prud'homme [22]. For relevant references on stabilized methods we refer to the subgrid viscosity method by Guermond [21], the orthogonal subscale method by Codina [14], the local projection method by Becker et al. [1,2,5] and the work on minimal stabilization procedures by Brezzi and Fortin [7].

The key issue in this paper is that the stabilization allows for estimates that are uniform in the Reynolds number. Hence the incompressible Euler equations are covered by the analysis. It is interesting to note that the present stabilized method allows for a complete decoupling of the analysis for the velocities and pressures. The only requirement for convergence is that the solution is sufficiently smooth, in a sense that will be detailed later, but most importantly we assume that the velocities $\mathbf{u} \in$ $\left[L^{2}\left(0, T ; H^{\frac{3}{2}+\epsilon}(\Omega)\right) \cap L^{\infty}\left(0, T ; W^{1, \infty}(\Omega)\right) \cap H^{1}\left(0, T ; L^{2}(\Omega)\right)\right]^{d}$ and the pressure $p \in L^{2}\left(0, T ; H^{\frac{1}{2}+\epsilon}(\Omega)\right)$. In case the solution has sufficient additional regularity we obtain the quasi-optimal error estimate for the velocity approximation:

$$
\left\|\mathbf{u}-\mathbf{u}_{h}\right\|_{L^{\infty}\left(0, T ; L^{2}(\Omega)\right)} \leq C h^{k+\frac{1}{2}}\|(\mathbf{u}, p)\|_{L^{2}\left(0, T ; H^{k+1}(\Omega)\right)},
$$

where $k$ denotes the polynomial order.

Our analysis is inspired by the one by Hansbo and Szepessy reported in [23], but our results using interior penalty stabilization are sharper. In fact, to control the convective velocity, which is only weakly divergence free, special non-linear stabilization terms are introduced in [23], leading to a more complex formulation and stronger regularity assumptions on the exact solution are required. In our case, the fact that the stabilization of the velocities is decoupled from the stabilization of the pressure allows us to prove convergence using essentially the stabilization terms of the linear case (see [10]), and under similar regularity assumptions. Moreover, we prove convergence for 
all polynomial orders, whereas in [23] the analysis was restricted to piecewise linear approximations in space and in time.

In this work we only consider discretization in space. For the sake of simplicity we assume the fluid velocity to be dimensionless and that the mean fluid velocity equals one. As a result the local Reynolds number is given by $\frac{h}{v}$. Focus will be put entirely on the convergence in the high Reynolds number regime $(v<h)$. The estimates are of course still valid in the low Reynolds number regime $(h<v)$, but then the regularity hypothesis may be relaxed while keeping optimal convergence if the stabilization parameters are properly chosen, see [10].

In the next section we introduce standard notation for the Navier-Stokes equations and briefly discuss the regularity assumptions. The stabilized finite element scheme, based on an interior penalty formulation, is introduced in Sect. 3. Some useful standard estimates are stated in Sect. 4. In Sect. 5, we study the wellposedness of the discrete scheme and its stability properties. The convergence analysis of the method is carried out in Sect. 6. First we prove convergence for the velocity and then for the pressure. The later requires an estimation of the error in the approximate acceleration. Finally, some conclusions are given in Sect. 7.

\section{The time-dependent Navier-Stokes equations}

Let $\Omega$ be a Lipschitz-continuous domain in $\mathbb{R}^{d}(d=2$ or 3 ) with a polyhedral boundary $\partial \Omega$ and outward pointing normal $\mathbf{n}$. For $T>0$ we consider the problem of solving, for $\mathbf{u}: \Omega \times(0, T) \longrightarrow \mathbb{R}^{d}$ and $p: \Omega \times(0, T) \longrightarrow \mathbb{R}$, the time-dependent incompressible Navier-Stokes equations with homogeneous boundary conditions:

$$
\left\{\begin{aligned}
\partial_{t} \mathbf{u}+\mathbf{u} \cdot \nabla \mathbf{u}-2 \nu \nabla \cdot \boldsymbol{\varepsilon}(\mathbf{u})+\nabla p & =\mathbf{f} \quad \text { in } \quad \Omega \times(0, T), \\
\nabla \cdot \mathbf{u} & =0 \quad \text { in } \Omega \times(0, T), \\
\mathbf{u} & =0 \quad \text { on } \partial \Omega \times(0, T), \\
\mathbf{u}(\cdot, 0) & =\mathbf{u}_{0} \quad \text { in } \Omega .
\end{aligned}\right.
$$

These equations describe the motion of a viscous incompressible fluid confined in $\Omega$. In (1), $v>0$ corresponds to the kinematic fluid viscosity coefficient, $\mathbf{f}: \Omega \times(0, T) \longrightarrow$ $\mathbb{R}^{d}$ represents a given source term, $\mathbf{u}_{0}: \Omega \longrightarrow \mathbb{R}^{d}$ stands for the initial velocity and

$$
\boldsymbol{\varepsilon}(\mathbf{u}) \stackrel{\text { def }}{=} \frac{1}{2}\left[\nabla \mathbf{u}+(\nabla \mathbf{u})^{\mathrm{T}}\right]
$$

for the strain rate tensor.

In the following, we will consider the usual Sobolev spaces $W^{m, q}(\Omega)$, with norm $\|\cdot\|_{m, q, \Omega}, m \geq 0$ and $q \geq 1$. In particular, we have $L^{q}(\Omega)=W^{0, q}(\Omega)$. We use the standard notation $H^{m}(\Omega) \stackrel{\text { def }}{=} W^{m, 2}(\Omega)$. The norm of $H^{m}(\Omega)$ is denoted by $\|\cdot\|_{m, \Omega}$ and its semi-norm by $|\cdot|_{m, \Omega}$. The space of $L^{2}(\Omega)$ divergence free functions is denoted by $H_{0}(\operatorname{div} ; \Omega)$. The scalar product in $L^{2}(\Omega)$ is denoted by $(\cdot, \cdot)$ and its norm by $\|\cdot\|_{0, \Omega}$. The closed subspaces $H_{0}^{1}(\Omega)$, consisting of functions in $H^{1}(\Omega)$ with zero trace on $\partial \Omega$, and $L_{0}^{2}(\Omega)$, consisting of function in $L^{2}(\Omega)$ with zero mean in $\Omega$, will also be used. 
Let us assume that the given functions $\mathbf{f}$ and $\mathbf{u}_{0}$ have, at least, the following regularity properties

$$
\mathbf{f} \in L^{\infty}\left(0, T ;\left[L^{2}(\Omega)\right]^{d}\right), \quad \mathbf{u}_{0} \in\left[L^{2}(\Omega)\right]^{d} .
$$

For sufficiently regular functions $\mathbf{u}$ and $p$, problem (1) holds if and only if

$$
\left\{\begin{aligned}
\left(\partial_{t} \mathbf{u}, \mathbf{v}\right)+c(\mathbf{u} ; \mathbf{u}, \mathbf{v})+a(\mathbf{u}, \mathbf{v})+b(p, \mathbf{v})=(\mathbf{f}, \mathbf{v}), & \text { a.e. in } \quad(0, T), \\
b(q, \mathbf{u})=0, & \text { a.e. in }(0, T), \\
\mathbf{u}(0)=\mathbf{u}_{0}, & \text { a.e. in } \Omega,
\end{aligned}\right.
$$

for all $(\mathbf{v}, q) \in\left[H_{0}^{1}(\Omega)\right]^{d} \times L_{0}^{2}(\Omega)$, and where

$$
\begin{aligned}
c(\mathbf{w} ; \mathbf{u}, \mathbf{v}) & \stackrel{\text { def }}{=}(\mathbf{w} \cdot \nabla \mathbf{u}, \mathbf{v}), \\
a(\mathbf{u}, \mathbf{v}) & \stackrel{\text { def }}{=} 2(v \boldsymbol{\varepsilon}(\mathbf{u}), \boldsymbol{\varepsilon}(\mathbf{v})), \\
b(p, \mathbf{v}) & \stackrel{\text { def }}{=}-(p, \nabla \cdot \mathbf{v}) .
\end{aligned}
$$

\subsection{Regularity assumptions}

For the analysis below to make sense, the solution and initial data must have the minimal regularity

$$
\begin{aligned}
& \mathbf{u} \in\left[L^{2}\left(0, T ; H^{\frac{3}{2}+\epsilon}(\Omega)\right) \cap L^{\infty}\left(0, T ; W^{1, \infty}(\Omega)\right) \cap H^{1}\left(0, T ; L^{2}(\Omega)\right)\right]^{d}, \\
& p \in L^{2}\left(0, T ; H^{\frac{1}{2}+\epsilon}(\Omega)\right), \quad \mathbf{u}_{0} \in\left[H^{\frac{3}{2}+\epsilon}(\Omega) \cap H_{0}^{1}(\Omega)\right]^{d} \cap H_{0}(\operatorname{div} ; \Omega) .
\end{aligned}
$$

In this paper we will for simplicity make the stronger regularity assumption

$$
\begin{aligned}
& \mathbf{u} \in\left[L^{\infty}\left(0, T ; W^{1, \infty}(\Omega)\right) \cap H^{1}\left(0, T ; L^{2}(\Omega)\right) \cap L^{\infty}\left(0, T ; H^{r}(\Omega)\right)\right]^{d}, \\
& p \in L^{2}\left(0, T ; H^{s}(\Omega)\right), \quad \mathbf{u}_{0} \in\left[H^{r}(\Omega) \cap H_{0}^{1}(\Omega)\right]^{d} \cap H_{0}(\operatorname{div} ; \Omega) .
\end{aligned}
$$

with $r, s \geq 2$, in order to use approximability and get optimal order estimates for the velocity.

Our pressure error estimates are bounded by the $L^{2}$-norm of the error in the approximate acceleration $\partial_{t} \mathbf{u}_{h}$. The error estimate we provide for this quantity requires the following additional regularity

$$
\begin{aligned}
& \mathbf{u} \in\left[H^{1}\left(0, T ; H^{r}(\Omega)\right)\right]^{d}, \\
& p \in L^{2}\left(0, T ; H^{s}(\Omega)\right) \cap H^{1}\left(0, T ; H^{1}(\Omega)\right) .
\end{aligned}
$$




\section{Space semi-discretization}

In this section we introduce a finite element discretization of problem (2) based on a weakly consistent interior penalty formulation with equal-order interpolations.

\subsection{Preliminaries}

Let $\left\{\mathcal{T}_{h}\right\}_{0<h \leq 1}$ be a family of triangulations of the domain $\Omega$ without hanging nodes. For each triangulation $\mathcal{T}_{h}$, the subscript $h \in(0,1]$ refers to the level of refinement of the triangulation, which is defined by

$$
h \stackrel{\text { def }}{=} \max _{K \in \mathcal{T}_{h}} h_{K}, \quad h_{K} \stackrel{\text { def }}{=} \max _{e \subset \partial K} h_{e},
$$

with $h_{e}$ the diameter of the face $e$.

Moreover we will assume that the family of triangulation $\left\{\mathcal{T}_{h}\right\}_{0<h \leq 1}$ is quasiuniform, i.e.,

$$
\frac{h_{K}}{\rho_{K}}<C_{\mathrm{R}}, \quad h_{K} \geq C_{\mathrm{U}} h, \quad \forall K \in \mathcal{T}_{h}, \quad \forall h \in(0,1],
$$

where $\rho_{K}$ stands for the diameter of the largest inscribed ball in $K$ and $C_{\mathrm{R}}, C_{\mathrm{U}}>0$ are fixed constants.

In what follows, the word faces refers to edges in $2 \mathrm{D}$ and faces in $3 \mathrm{D}$, and the distinction will not be made unless necessary. For a given piecewise continuous function $\varphi$, the jump $\llbracket \varphi \rrbracket_{e}$ over a face $e$ is defined by

$$
\llbracket \varphi \rrbracket_{e}(\mathbf{x}) \stackrel{\text { def }}{=}\left\{\begin{aligned}
\lim _{t \rightarrow 0^{+}}\left(\varphi\left(\mathbf{x}-t \mathbf{n}_{e}\right)-\varphi\left(\mathbf{x}+t \mathbf{n}_{e}\right)\right), & \text { if } e \not \subset \partial \Omega, \\
0, & \text { if } e \subset \partial \Omega,
\end{aligned}\right.
$$

where $\mathbf{n}_{e}$ is a fixed but arbitrary normal unit vector on $e$ and $\mathbf{x} \in e$.

In this paper, we let $V_{h}^{k}$ denote the standard space of continuous piecewise polynomial functions of degree $k \geq 1$,

$$
V_{h}^{k} \stackrel{\text { def }}{=}\left\{v \in H^{1}(\Omega): v_{\mid K} \in \mathbb{P}_{k}(K), \quad \forall K \in \mathcal{T}_{h}\right\}
$$

and $H^{2}\left(\mathcal{T}_{h}\right)$ the space of piecewise $H^{2}$ functions,

$$
H^{2}\left(\mathcal{T}_{h}\right) \stackrel{\text { def }}{=}\left\{v: \Omega \longrightarrow \mathbb{R}: v_{\mid K} \in H^{2}(K), \quad \forall K \in \mathcal{T}_{h}\right\}
$$

For the velocities we will use the space $\left[V_{h}^{k}\right]^{d}$ and for the pressure we will use $Q_{h}^{k} \stackrel{\text { def }}{=}$ $V_{h}^{k} \cap L_{0}^{2}(\Omega)$. 
3.2 An interior penalty finite element method

Denoting the product space $W_{h}^{k} \stackrel{\text { def }}{=}\left[V_{h}^{k}\right]^{d} \times Q_{h}^{k}$ our space semi-discretized scheme reads: for all $t \in(0, T)$, find $\left(\mathbf{u}_{h}(t), p_{h}(t)\right) \in W_{h}^{k}$ such that

$$
\begin{aligned}
\left(\partial_{t} \mathbf{u}_{h}, \mathbf{v}_{h}\right)+(\mathbf{A}+\mathbf{J})\left[\mathbf{u}_{h} ;\left(\mathbf{u}_{h}, p_{h}\right),\left(\mathbf{v}_{h}, q_{h}\right)\right] & =\left(\mathbf{f}, \mathbf{v}_{h}\right), \\
\mathbf{u}_{h}(0) & =\mathbf{u}_{0, h},
\end{aligned}
$$

for all $\left(\mathbf{v}_{h}, q_{h}\right) \in W_{h}^{k}$ and with $\mathbf{u}_{0, h}$ a suitable approximation of $\mathbf{u}_{0}$ in $\left[V_{h}^{k}\right]^{d}$. In (9) we used the following notations:

$$
\begin{aligned}
\mathbf{A}\left[\mathbf{w}_{h} ;\left(\mathbf{u}_{h}, p_{h}\right),\left(\mathbf{v}_{h}, q_{h}\right)\right] \stackrel{\text { def }}{=} & a_{h}\left(\mathbf{u}_{h}, \mathbf{v}_{h}\right)+c_{h}\left(\mathbf{w}_{h} ; \mathbf{u}_{h}, \mathbf{v}_{h}\right) \\
& +b_{h}\left(p_{h}, \mathbf{v}_{h}\right)-b_{h}\left(q_{h}, \mathbf{u}_{h}\right) \\
c_{h}\left(\mathbf{w}_{h} ; \mathbf{u}_{h}, \mathbf{v}_{h}\right) \stackrel{\text { def }}{=} & c\left(\mathbf{w}_{h} ; \mathbf{u}_{h}, \mathbf{v}_{h}\right)+\frac{1}{2}\left(\nabla \cdot \mathbf{w}_{h} \mathbf{u}_{h}, \mathbf{v}_{h}\right) \\
& -\frac{1}{2}\left\langle\mathbf{w}_{h} \cdot \mathbf{n} \mathbf{u}_{h}, \mathbf{v}_{h}\right\rangle_{\partial \Omega} \\
a_{h}\left(\mathbf{u}_{h}, \mathbf{v}_{h}\right) \stackrel{\text { def }}{=} & a\left(\mathbf{u}_{h}, \mathbf{v}_{h}\right)-\left\langle 2 v \boldsymbol{\varepsilon}\left(\mathbf{u}_{h}\right) \mathbf{n}, \mathbf{v}_{h}\right\rangle_{\partial \Omega}-\left\langle\mathbf{u}_{h}, 2 v \boldsymbol{\varepsilon}\left(\mathbf{v}_{h}\right) \mathbf{n}\right\rangle_{\partial \Omega} \\
& +\left\langle\gamma_{v} \frac{v}{h} \mathbf{u}_{h}, \mathbf{v}_{h}\right\rangle_{\partial \Omega}+\left\langle\mathbf{u}_{h} \cdot \mathbf{n}, \mathbf{v}_{h} \cdot \mathbf{n}\right\rangle_{\partial \Omega} \\
\mathbf{J}\left[\mathbf{w}_{h} ;\left(\mathbf{u}_{h}, p_{h}\right),\left(\mathbf{v}_{h}, q_{h}\right)\right] \stackrel{\text { def }}{=} & j_{\mathbf{w}_{h}}\left(\mathbf{u}_{h}, \mathbf{v}_{h}\right)+\gamma j\left(\mathbf{u}_{h}, \mathbf{v}_{h}\right) \\
& +j\left(p_{h}, q_{h}\right)
\end{aligned}
$$

with

$$
\begin{aligned}
j_{\mathbf{w}_{h}}\left(\mathbf{u}_{h}, \mathbf{v}_{h}\right) & \stackrel{\text { def }}{=} \sum_{K \in \mathcal{T}_{h}{ }_{\partial}} \int_{K} h_{K}^{2}\left|\mathcal{I}_{h}^{1} \mathbf{w}_{h} \cdot \mathbf{n}\right|^{2} \llbracket \nabla \mathbf{u}_{h} \rrbracket: \llbracket \nabla \mathbf{v}_{h} \rrbracket \mathrm{d} \boldsymbol{s}, \\
j\left(\mathbf{u}_{h}, \mathbf{v}_{h}\right) & \stackrel{\text { def }}{=} \sum_{K \in \mathcal{T}_{h}{ }_{\partial K}} h_{K}^{2} \llbracket \nabla \mathbf{u}_{h} \rrbracket: \llbracket \nabla \mathbf{v}_{h} \rrbracket \mathrm{d} \boldsymbol{s}, \\
j\left(p_{h}, q_{h}\right) & \stackrel{\text { def }}{=} \sum_{K \in \mathcal{T}_{h} \int_{K}} h_{K}^{2} \llbracket \nabla p_{h} \rrbracket \cdot \llbracket \nabla q_{h} \rrbracket \mathrm{d} \boldsymbol{s} .
\end{aligned}
$$

Here, $\mathcal{I}_{h}^{1} \mathbf{w}_{h}$ denotes the interpolation of $\mathbf{w}_{h}$ onto the space $\left[V_{h}^{1}\right]^{d}$ (continuous piecewise linear) and $\gamma, \gamma_{\nu}$ two positive constants to be fixed later on.

Some remarks are in order. We point out that the additional terms appearing in the discrete bilinear form $\mathbf{A}$, compared to the formulation (2), are due to the non satisfaction of the divergence free condition and to the weakly imposed boundary conditions of Nitsche type. To counter effects of insufficient control of the divergence free condition, an artificial term is added that ensures coercivity while remaining strongly consistent [33] (since $\nabla \cdot \mathbf{u}=0$ for the exact solution). The Nitsche type boundary 
conditions are inspired by those analyzed in [10] and [19]. In the stabilization term $j_{\mathbf{w}_{h}}(\cdot, \cdot)$ we use the $\mathbb{P}_{1}$-interpolant of the velocity vector $\mathbf{w}_{h}$ as weight. This may be replaced by the function $\mathbf{w}_{h}$ itself or the max value of $\mathbf{w}_{h}$ on the face depending on what is most convenient from implementation standpoint. The analysis below carries over to these versions with minor modifications.

The discrete formulation (9) satisfies the following approximate Galerkin Orthogonality.

Lemma 1 (Approximate Galerkin Orthogonality) Let $(\mathbf{u}, p)$ be the solution of (1), $\left(\mathbf{u}_{h}, p_{h}\right) \in W_{h}^{k}$ the solution of (9) and assume that $(\mathbf{u}, p)$ has the minimal regularity (4). Then,

$$
\begin{aligned}
& \left(\partial_{t}\left(\mathbf{u}-\mathbf{u}_{h}\right), \mathbf{v}_{h}\right)+\mathbf{A}\left[\mathbf{u} ;(\mathbf{u}, p),\left(\mathbf{v}_{h}, q_{h}\right)\right] \\
& \quad-(\mathbf{A}+\mathbf{J})\left[\mathbf{u}_{h},\left(\mathbf{u}_{h}, p_{h}\right),\left(\mathbf{v}_{h}, q_{h}\right)\right]=0, \text { a.e. in }(0, T),
\end{aligned}
$$

for all $\left(\mathbf{v}_{h}, q_{h}\right) \in W_{h}^{k}$.

Proof This is an immediate consequence of the consistency of the standard Galerkin method.

\section{Interpolation}

In this section we shall state some standard estimates that will be useful for the convergence analysis below. First, we recall the following local inverse estimate (see [18, page 75], for instance): for all $v_{h} \in V_{h}^{k}$, and $K \in \mathcal{T}_{h}, 0<h \leq 1$, there holds

$$
\left\|v_{h}\right\|_{l, p, K} \leq C_{\mathrm{I}} h_{K}^{m-l+d\left(\frac{1}{p}-\frac{1}{q}\right)}\left\|v_{h}\right\|_{m, q, K}
$$

with $C_{\text {I }}$ a positive constant, independent of $h, K, p$ and $q$, and where $0 \leq m \leq l$ and $1 \leq p, q \leq \infty$.

Let $\Pi_{h}^{k}$ and $\mathcal{I}_{h}^{k}$ be, respectively, the $L^{2}$-projection and the Lagrange interpolant on $V_{h}^{k}$. For $u \in H^{r}(\Omega), r \geq 2$, we have the following standard error estimate (see [18], for instance),

$$
\left\|\mathcal{I}_{h}^{k} u-u\right\|_{0, \Omega}+h\left\|\nabla\left(\mathcal{I}_{h}^{k} u-u\right)\right\|_{0, \Omega} \leq C h^{r_{\mathbf{u}}}\|u\|_{r_{\mathbf{u}}, \Omega},
$$

where $r_{\mathbf{u}} \stackrel{\text { def }}{=} \min \{r, k+1\}$. The following stability estimates for the $L^{2}$-projection hold,

$$
\begin{aligned}
\left\|\Pi_{h}^{k} u\right\|_{0, \Omega} & \leq C\|u\|_{0, \Omega}, \\
\left\|\Pi_{h}^{k} u\right\|_{1, \Omega} & \leq C\|u\|_{1, \Omega},
\end{aligned}
$$

for all $u \in H^{1}(\Omega)$. Thus, from (17), we then deduce that

$$
\left\|u-\Pi_{h}^{k} u\right\|_{0, \Omega}+h\left\|\nabla\left(u-\Pi_{h}^{k} u\right)\right\|_{0, \Omega} \leq C h^{r_{\mathbf{u}}}\|u\|_{r_{\mathbf{u}}, \Omega},
$$


for $u \in H^{r}(\Omega)$. In addition, the following stability result holds where $C_{\pi}>0$ is a constant independent of $h$ (but not of the polynomial order),

$$
\begin{aligned}
& \left\|\Pi_{h}^{k} u\right\|_{0, \infty, \Omega} \leq C_{\pi}\|u\|_{0, \infty, \Omega}, \quad \forall u \in L^{\infty}(\Omega), \\
& \left\|\Pi_{h}^{k} u\right\|_{1, \infty, \Omega} \leq C_{\pi}\|u\|_{1, \infty, \Omega}, \quad \forall u \in W^{1, \infty}(\Omega) .
\end{aligned}
$$

The second estimate easily follows from the first noting that, from (16), we have

$$
\begin{aligned}
\left\|\nabla \Pi_{h}^{k} u\right\|_{0, \infty, \Omega} & =\left\|\nabla\left(\Pi_{h}^{k} u-\Pi_{\tilde{K}}^{0} u\right)\right\|_{0, \infty, \tilde{K}} \\
& \leq C h^{-1}\left\|\Pi_{h}^{k} u-\Pi_{\tilde{K}}^{0} u\right\|_{0, \infty, \tilde{K}} \\
& \leq C h^{-1}\left(\left\|\Pi_{h}^{k} u-u\right\|_{0, \infty, \Omega}+\left\|u-\Pi_{\tilde{K}}^{0} u\right\|_{0, \infty, \tilde{K}}\right) \\
& \leq C h^{-1}\left(\left\|\mathcal{I}_{h}^{k} u-u\right\|_{0, \infty, \Omega}+\left\|u-\Pi_{\tilde{K}}^{0} u\right\|_{0, \infty, \tilde{K}}\right),
\end{aligned}
$$

where $\tilde{K} \in \mathcal{T}_{h}$ stands for the element where the maximum value is taken, and $\Pi_{\tilde{K}}^{0} u$ denotes the $L^{2}$-projection of $u$ onto a piecewise constant on $\tilde{K}$. Applying now (20) to the first term of the right hand side we conclude

$$
\begin{aligned}
\left\|\nabla \Pi_{h}^{k} u\right\|_{0, \infty, \Omega} & \leq C h^{-1}\left(\left\|\mathcal{I}_{h}^{k} u-u\right\|_{0, \infty, \Omega}+\left\|u-\Pi_{\tilde{K}}^{0} u\right\|_{0, \infty, \tilde{K}}\right) \\
& \leq C\|\nabla u\|_{0, \infty, \Omega} .
\end{aligned}
$$

It then follows that

$$
\left\|\Pi_{h}^{k} u-u\right\|_{0, \infty, \Omega}+h\left\|\Pi_{h}^{k} u-u\right\|_{1, \infty, \Omega} \leq C h\|u\|_{1, \infty, \Omega},
$$

for all $u \in W^{1, \infty}(\Omega)$.

The results (20) and (21) have been proved in $[4,6,16]$ for low order elements. We would like to point to the last reference, which readily extends this results to higher order elements and which gives weighted estimates. Using these estimates, the assumption of mesh quasi-uniformity in the present paper may be relaxed to local quasi-uniformity.

In order to handle the non-linear terms, we shall also need a discrete commutator property, which is stated in the following lemma (for a proof, see [3,29]).

Lemma 2 Let $\mathcal{S Z}_{h}^{k}: W^{1, \infty}(\Omega) \rightarrow V_{h}^{k}$ denote the Scott-Zhang interpolator [32]. There exists a constant $C_{\mathrm{B}}>0$ independent of $h$, such that for all $u \in W^{1, \infty}(\Omega)$ and $v_{h} \in V_{h}^{k}$,

$$
\left\|\mathcal{S} \mathcal{Z}_{h}^{k}\left(u v_{h}\right)-u v_{h}\right\|_{0, \Omega} \leq C_{B} h\|u\|_{1, \infty, \Omega}\left\|v_{h}\right\|_{0, \Omega} .
$$

The following corollary is a direct consequence of the previous result. 
Corollary 1 For all $u \in W^{1, \infty}(\Omega)$ and $v_{h} \in V_{h}^{k}$ there holds

$$
\begin{aligned}
& \left\|\Pi_{h}^{k}\left(u v_{h}\right)-u v_{h}\right\|_{0, \Omega} \leq h\|u\|_{1, \infty, \Omega}\left\|v_{h}\right\|_{0, \Omega}, \\
& \left\|\Pi_{h}^{k}\left(u v_{h}\right)-\Pi_{h}^{k} u v_{h}\right\|_{0, \Omega} \leq h\|u\|_{1, \infty, \Omega}\left\|v_{h}\right\|_{0, \Omega} .
\end{aligned}
$$

For the error analysis, we shall also use the trace inequality

$$
\|v\|_{0, \partial K}^{2} \leq C\left(h_{K}^{-1}\|v\|_{0, K}^{2}+h_{K}\|\nabla v\|_{0, K}^{2}\right), \quad \forall v \in H^{1}(K),
$$

see [13] (or [34] for a detailed proof). In particular, by combining the above estimate with the inverse inequality (16), it follows that

$$
\left\|v_{h}\right\|_{0, \partial K}^{2} \leq C_{\mathrm{T}} h_{K}^{-1}\left\|v_{h}\right\|_{0, K}^{2}, \quad \forall v_{h} \in V_{h}^{k}
$$

The uniform (in $v$ ) stability of the present method relies on the fact that the gradient jumps in (9) can control some interpolation errors of the stream-line derivative, divergence and pressure gradient. This is formalized, in the following lemma, by establishing some error bounds for the Oswald quasi-interpolant $\pi_{h}^{*}$ (see $\left.[28,31]\right)$.

Definition 1 For each node $x_{i}$, let $n_{i}$ be the number of elements containing $x_{i}$ as a node. We define a quasi-interpolant $\pi_{h}^{*}$ of degree $k$ by

$$
\pi_{h}^{*} v\left(x_{i}\right) \stackrel{\text { def }}{=} \frac{1}{n_{i}} \sum_{\left\{K: x_{i} \in K\right\}} v_{\mid K}\left(x_{i}\right), \quad \forall v \in\left[H^{2}\left(\mathcal{T}_{h}\right)\right]^{d}
$$

with $H^{2}\left(\mathcal{T}_{h}\right)$ given by (8).

Lemma 3 There exist three constants $\gamma_{i}>0, i=1,2,3$, depending on the local mesh geometry, but not on the mesh size $h$, such that

$$
\begin{aligned}
\left\|h^{\frac{1}{2}}\left(\mathbf{w}_{h} \cdot \nabla \mathbf{v}_{h}-\pi_{h}^{*}\left(\mathbf{w}_{h} \cdot \nabla \mathbf{v}_{h}\right)\right)\right\|_{0, \Omega}^{2} & \leq \gamma_{1} j_{\mathbf{w}_{h}}\left(\mathbf{v}_{h}, \mathbf{v}_{h}\right), \\
\left\|h^{\frac{1}{2}}\left(\nabla \cdot \mathbf{v}_{h}-\pi_{h}^{*}\left(\nabla \cdot \mathbf{v}_{h}\right)\right)\right\|_{0, \Omega}^{2} & \leq \gamma_{2} j\left(\mathbf{v}_{h}, \mathbf{v}_{h}\right), \\
\left\|h^{\frac{1}{2}}\left(\nabla q_{h}-\pi_{h}^{*}\left(\nabla q_{h}\right)\right)\right\|_{0, \Omega}^{2} & \leq \gamma_{3} j\left(q_{h}, q_{h}\right),
\end{aligned}
$$

for all $\left(\mathbf{v}_{h}, q_{h}, \mathbf{w}_{h}\right) \in\left[V_{h}^{k}\right]^{d} \times V_{h}^{k} \times\left[V_{h}^{1}\right]^{d}$.

Proof A proof of (26)-(28) can be found in $[8,10]$.

We introduce now, for each $\mathbf{w}_{h} \in\left[V_{h}^{k}\right]^{d}$ given, the triple-norm

$$
\left\|\left(\mathbf{v}_{h}, q_{h}\right)\right\|_{\mathbf{w}_{h}}^{2} \stackrel{\text { def }}{=}\left\|\mathbf{v}_{h}\right\|^{2}+\mathbf{J}\left[\mathbf{w}_{h} ;\left(\mathbf{v}_{h}, q_{h}\right),\left(\mathbf{v}_{h}, q_{h}\right)\right]
$$


with

$$
\begin{aligned}
\left\|\mathbf{v}_{h}\right\|^{2} \stackrel{\text { def }}{=} & \left\|v^{\frac{1}{2}} \nabla \mathbf{v}_{h}\right\|_{0, \Omega}^{2}+\left\|h^{\frac{1}{2}} \nabla \cdot \mathbf{v}_{h}\right\|_{0, \Omega}^{2}+\left\|\left(\gamma_{\nu} v\right)^{\frac{1}{2}} h^{-\frac{1}{2}} \mathbf{v}_{h}\right\|_{0, \partial \Omega}^{2} \\
& +\left\|\mathbf{v}_{h} \cdot \mathbf{n}\right\|_{0, \partial \Omega}^{2} .
\end{aligned}
$$

For the continuity of the Stokes system, with Nitsche boundary conditions, it is also convenient to introduce a norm valid for functions $(\mathbf{v}, q) \in\left[H^{\frac{3}{2}+\epsilon}(\Omega)\right]^{d} \times H^{\frac{1}{2}+\epsilon}(\Omega)$,

$$
\begin{aligned}
\|(\mathbf{v}, q) \rrbracket\|^{2} \stackrel{\text { def }}{=} & \left\|v^{\frac{1}{2}} \nabla \mathbf{v}\right\|_{0, \Omega}^{2}+\left\|h^{\frac{1}{2}} \nabla \cdot \mathbf{v}\right\|_{0, \Omega}^{2}+\left\|h^{-\frac{1}{2}} q\right\|_{0, \Omega}^{2} \\
& +\left\|h^{-\frac{1}{2}} \mathbf{v}\right\|_{0, \Omega}^{2}+\left\|(v h)^{\frac{1}{2}} \nabla \mathbf{v}\right\|_{0, \partial \Omega}^{2}+\|q\|_{0, \partial \Omega}^{2} .
\end{aligned}
$$

For these two norms we have the following approximation result.

Lemma 4 Assume that (5) holds. Then we have

$$
\begin{aligned}
\left\|\left(\mathbf{u}-\Pi_{h}^{k} \mathbf{u}, p-\Pi_{h}^{k} p\right)\right\|_{\mathbf{0}} \leq & C\left(v^{\frac{1}{2}}+h^{\frac{1}{2}}\right) h^{r_{\mathbf{u}}-1}\|\mathbf{u}\|_{r_{\mathbf{u}}, \Omega} \\
& +C h^{r_{p}-\frac{1}{2}}\|p\|_{r_{p}, \Omega}
\end{aligned}
$$

and

$$
\begin{aligned}
\|\left(\mathbf{u}-\Pi_{h}^{k} \mathbf{u}, p-\Pi_{h}^{k} p\right) \rrbracket \leq & C\left(v^{\frac{1}{2}}+h^{\frac{1}{2}}\right) h^{r_{\mathbf{u}}-1}\|\mathbf{u}\|_{r_{\mathbf{u}}, \Omega} \\
& +C h^{r_{p}-\frac{1}{2}}\|p\|_{r_{p}, \Omega},
\end{aligned}
$$

with $r_{\mathbf{u}} \stackrel{\text { def }}{=} \min \{r, k+1\}$ and $r_{p} \stackrel{\text { def }}{=} \min \{s, k+1\}, C>0$ a constant depending only on $\gamma_{v}, \gamma$.

Proof From (19) we have

$$
\left\|v^{\frac{1}{2}} \nabla\left(\mathbf{u}-\Pi_{h}^{k} \mathbf{u}\right)\right\|_{0, \Omega}^{2} \leq C \nu h^{2\left(r_{\mathbf{u}}-1\right)}\|\mathbf{u}\|_{r_{\mathbf{u}}, \Omega}^{2},
$$

and

$$
\left\|h^{\frac{1}{2}} \nabla \cdot\left(\mathbf{u}-\Pi_{h}^{k} \mathbf{u}\right)\right\|_{0, \Omega}^{2} \leq C h^{2 r_{\mathbf{u}}-1}\|\mathbf{u}\|_{r_{\mathbf{u}}, \Omega}^{2}
$$

We treat the boundary terms using the trace inequality (24) in combination with (19) and the quasi-uniformity of the triangulation (7), yielding

$$
\begin{aligned}
\left\|\mathbf{u}-\Pi_{h}^{k} \mathbf{u}\right\|_{0, \partial \Omega}^{2} \leq & C \sum_{e \subset \partial \Omega}\left(h_{K_{e}}^{-1}\left\|\mathbf{u}-\Pi_{h}^{k} \mathbf{u}\right\|_{0, K_{e}}^{2}\right. \\
& \left.+h_{K_{e}}\left\|\nabla\left(\mathbf{u}-\Pi_{h}^{k} \mathbf{u}\right)\right\|_{0, K_{e}}^{2}\right) \\
\leq & C h^{2 r_{\mathbf{u}}-1}\|\mathbf{u}\|_{r_{\mathbf{u}}, \Omega}^{2},
\end{aligned}
$$


where $K_{e}$ denotes the simplex such that $e \subset \partial K_{e} \cap \partial \Omega$. The interior penalty terms are treated in the same fashion as the boundary terms. We have

$$
\begin{aligned}
& j\left(\mathbf{u}-\Pi_{h}^{k} \mathbf{u}, \mathbf{u}-\Pi_{h}^{k} \mathbf{u}\right)=\sum_{K \in \mathcal{T}_{h}} h_{K}^{2} \int_{\partial K} \llbracket \nabla\left(\mathbf{u}-\Pi_{h}^{k} \mathbf{u}\right) \rrbracket^{2} \mathrm{~d} \mathbf{s} \\
& \leq C \sum_{K \in \mathcal{T}_{h}} h_{K}^{2}\left\|\nabla\left(\mathbf{u}-\Pi_{h}^{k} \mathbf{u}\right)\right\|_{0, \partial K}^{2} \\
& \leq C \sum_{K \in \mathcal{T}_{h}}\left(h_{K}\left\|\nabla\left(\mathbf{u}-\Pi_{h}^{k} \mathbf{u}\right)\right\|_{0, K}^{2}+h_{K}^{3}\left\|\nabla^{2}\left(\mathbf{u}-\Pi_{h}^{k} \mathbf{u}\right)\right\|_{0, K}^{2}\right) \\
& \leq C\left(h\left\|\nabla\left(\mathbf{u}-\Pi_{h}^{k} \mathbf{u}\right)\right\|_{0, \Omega}^{2}+h^{3}\left\|\nabla^{2}\left(\mathbf{u}-\Pi_{h}^{k} \mathbf{u}\right)\right\|_{0, \Omega}^{2}\right) \\
& \leq C h^{2 r_{\mathbf{u}}-1}\|\mathbf{u}\|_{r_{\mathbf{u}}, \Omega}^{2} .
\end{aligned}
$$

Obviously, the pressure jump term is treated using the same argument, which completes the proof of (31).

To prove (32) we simply note that by trace inequalities and the stability of the $L^{2}$-projection there holds

$$
\begin{aligned}
\left\|(v h)^{\frac{1}{2}} \nabla\left(\mathbf{u}-\Pi_{h}^{k} \mathbf{u}\right)\right\|_{0, \partial \Omega}^{2} \leq & C\left\|v^{\frac{1}{2}} \nabla\left(\mathbf{u}-\mathcal{I}_{h}^{k} \mathbf{u}\right)\right\|_{0, \Omega}^{2} \\
& +C h^{2} \sum_{K \in \mathcal{T}_{h}}\left\|v^{\frac{1}{2}}\left(\mathbf{u}-\mathcal{I}_{h}^{k} \mathbf{u}\right)\right\|_{2, K}^{2} \\
\leq & C \nu h^{2\left(r_{\mathbf{u}}-1\right)}\|\mathbf{u}\|_{r_{\mathbf{u}}, \Omega}^{2} .
\end{aligned}
$$

To conclude, we apply the inequality (33) to the term $\left\|p-\Pi_{h}^{k} p\right\|_{0, \partial \Omega}^{2}$.

Finally, we shall also make use of the following projection operator, based on a Stokes-like problem. For each $\mathbf{u} \in\left[H^{\frac{3}{2}+\epsilon}(\Omega) \cap H_{0}^{1}(\Omega)\right]^{d} \cap H_{0}(\operatorname{div} ; \Omega)$, we denote by $S_{h}^{k} \mathbf{u} \stackrel{\text { def }}{=}\left(P_{h}^{k} \mathbf{u}, R_{h}^{k} \mathbf{u}\right) \in W_{h}^{k}$ the unique solution of

$$
\left\{\begin{array}{c}
\left(P_{h}^{k} \mathbf{u}, \mathbf{v}_{h}\right)+a_{h}\left(P_{h}^{k} \mathbf{u}, \mathbf{v}_{h}\right)+b_{h}\left(R_{h}^{k} \mathbf{u}, \mathbf{v}_{h}\right) \\
+\gamma j\left(P_{h}^{k} \mathbf{u}, \mathbf{v}_{h}\right)=\left(\mathbf{u}, \mathbf{v}_{h}\right)+a_{h}\left(\mathbf{u}, \mathbf{v}_{h}\right) \\
-b_{h}\left(q_{h}, P_{h}^{k} \mathbf{u}\right)+j\left(R_{h}^{k} \mathbf{u}, q_{h}\right)=0
\end{array}\right.
$$

for all $\left(\mathbf{v}_{h}, q_{h}\right) \in W_{h}^{k}$.

By assuming that $\mathbf{u}$ is also sufficiently regular in time, so that the projection makes sense at each time $t$, we have the following approximation result, whose proof is based on the results reported in [10]. 
Lemma 5 Let $\mathbf{u} \in\left[L^{\infty}\left(0, T ; H^{r}(\Omega) \cap H_{0}^{1}(\Omega)\right)\right]^{d} \cap H_{0}(\operatorname{div} ; \Omega)$. The following error estimate for the projection $P_{h}^{k}$ holds:

$$
\left\|\mathbf{u}-P_{h}^{k} \mathbf{u}\right\|_{L^{\infty}\left(0, T ; L^{2}(\Omega)\right)} \leq C\left(v^{\frac{1}{2}}+h^{\frac{1}{2}}\right) h^{r_{\mathbf{u}}-1}\|\mathbf{u}\|_{L^{\infty}\left(0, T ; H^{r} \mathbf{u}(\Omega)\right)} .
$$

Moreover, if in addition $\partial_{t} \mathbf{u} \in\left[L^{2}\left(0, T ; H^{r}(\Omega)\right)\right]^{d}$, we have

$$
\left\|\partial_{t}\left(\mathbf{u}-P_{h}^{k} \mathbf{u}\right)\right\|_{L^{2}\left(0, T ; L^{2}(\Omega)\right)} \leq C\left(v^{\frac{1}{2}}+h^{\frac{1}{2}}\right) h^{r_{\mathbf{u}}-1}\left\|\partial_{t} \mathbf{u}\right\|_{L^{2}\left(0, T ; H^{r} \mathbf{u}(\Omega)\right)} .
$$

With $r_{\mathbf{u}} \stackrel{\text { def }}{=} \min \{r, k+1\}$ and $C>0$ independent of $v$ and $h$.

\section{Stability}

In this section we investigate the wellposedness and some stability properties of the discrete scheme (9).

\subsection{Existence and uniqueness of discrete solution}

The following modified inf-sup condition states the stability of the discrete pressures.

Lemma 6 There exists two constants $C, \beta>0$, independent of $h$ and $v$, such that

$$
\sup _{\mathbf{v}_{h} \in\left[V_{h}^{k}\right]^{d}} \frac{\left|b_{h}\left(q_{h}, \mathbf{v}_{h}\right)\right|}{\left\|\mathbf{v}_{h}\right\|_{1, \Omega}}+C h^{\frac{1}{2}} j\left(q_{h}, q_{h}\right)^{\frac{1}{2}} \geq \beta\left\|q_{h}\right\|_{0, \Omega},
$$

for all $q_{h} \in Q_{h}^{k}$.

Proof Let $q_{h} \in Q_{n}^{k}$. From [20, Corollary 2.4], there exists $\mathbf{v}_{q} \in\left[H_{0}^{1}(\Omega)\right]^{d}$ such that

$$
\nabla \cdot \mathbf{v}_{q}=q_{h}, \quad\left\|\mathbf{v}_{q}\right\|_{1, \Omega} \leq C\left\|q_{h}\right\|_{0, \Omega} .
$$

Thus, using integration by parts and (13), we have

$$
\begin{aligned}
\left\|q_{h}\right\|_{0, \Omega}^{2}= & \left(q_{h}, \nabla \cdot \mathbf{v}_{q}\right) \\
= & \left(q_{h}, \nabla \cdot \mathbf{v}_{q}-\nabla \cdot \Pi_{h}^{k} \mathbf{v}_{q}\right)+\left(q_{h}, \nabla \cdot \Pi_{h}^{k} \mathbf{v}_{q}\right) \\
= & \left(\nabla q_{h}, \mathbf{v}_{q}-\Pi_{h}^{k} \mathbf{v}_{q}\right)-\left\langle q_{h},\left(\Pi_{h}^{k} \mathbf{v}_{q}\right) \cdot \mathbf{n}\right\rangle_{\partial \Omega} \\
& +\left(q_{h}, \nabla \cdot \Pi_{h}^{k} \mathbf{v}_{q}\right) \\
= & \left(\nabla q_{h}, \mathbf{v}_{q}-\Pi_{h}^{k} \mathbf{v}_{q}\right)-b_{h}\left(q_{h}, \Pi_{h}^{k} \mathbf{v}_{q}\right) .
\end{aligned}
$$


In particular, using the orthogonality of the $L^{2}$-projection, Cauchy-Schwarz inequality, Lemma 3 and (19), we get

$$
\begin{aligned}
\left|\left(\nabla q_{h}, \mathbf{v}_{q}-\Pi_{h}^{k} \mathbf{v}_{q}\right)\right| & =\left|\left(\nabla q_{h}-\Pi_{h}^{k}\left(\nabla q_{h}\right), \mathbf{v}_{q}-\Pi_{h}^{k} \mathbf{v}_{q}\right)\right| \\
& \leq\left\|\nabla q_{h}-\Pi_{h}^{k}\left(\nabla q_{h}\right)\right\|_{0, \Omega}\left\|\mathbf{v}_{q}-\Pi_{h}^{k} \mathbf{v}_{q}\right\|_{0, \Omega} \\
& \leq C h^{-\frac{1}{2}} j\left(q_{h}, q_{h}\right)^{\frac{1}{2}}\left\|\mathbf{v}_{q}-\Pi_{h}^{k} \mathbf{v}_{q}\right\|_{0, \Omega} \\
& \leq C^{\frac{1}{2}} j\left(q_{h}, q_{h}\right)^{\frac{1}{2}}\left\|\mathbf{v}_{q}\right\|_{1, \Omega} .
\end{aligned}
$$

Thus, from (37), if follows that

$$
\left|b_{h}\left(q_{h}, \Pi_{h}^{k} \mathbf{v}_{q}\right)\right|+C h^{\frac{1}{2}} j\left(q_{h}, q_{h}\right)^{\frac{1}{2}}\left\|\mathbf{v}_{q}\right\|_{1, \Omega} \geq\left\|q_{h}\right\|_{0, \Omega}^{2} .
$$

In addition, from (18) and (36), we have

$$
\begin{aligned}
\left\|\Pi_{h}^{k} \mathbf{v}_{q}\right\|_{1, \Omega} & \leq C\left\|\mathbf{v}_{q}\right\|_{1, \Omega} \\
& \leq C\left\|q_{h}\right\|_{0, \Omega}
\end{aligned}
$$

which completes the proof.

Remark 1 Note the factor $h^{\frac{1}{2}}$ in front of the stabilization operator in equation (35). This shows that the stabilization of the pressure may be relaxed while keeping a uniform inf-sup condition. In the low Reynolds number regime this observation may be used to obtain optimal convergence estimates when $\mathbf{u} \in H^{r}(\Omega)$ and $p \in H^{r-1}(\Omega), r \geq 2$. See [10] for further details.

At this point, it is worth introducing the following discrete pressure and velocity subspaces:

$$
\begin{aligned}
& C_{h, k}^{1} \stackrel{\text { def }}{=}\left\{q_{h} \in Q_{h}^{k}: j\left(q_{h}, q_{h}\right)=0\right\}, \\
& V_{h, k}^{\text {div }} \stackrel{\text { def }}{=}\left\{\mathbf{v}_{h} \in\left[V_{h}^{k}\right]^{d}: b_{h}\left(q_{h}, \mathbf{v}_{h}\right)=0, \quad \forall q_{h} \in C_{h, k}^{1}\right\} .
\end{aligned}
$$

Clearly $Q_{h}^{k} \cap C^{1}(\Omega) \subset C_{h, k}^{1}$, and since $\mathbb{P}_{k}(\Omega) \subset Q_{h}^{k} \cap C^{1}(\Omega)$, it then follows that $C_{h, k}^{1} \neq\{0\}$. On the other hand, next corollary (which is a direct consequence of Lemma 6) states that $V_{h, k}^{\text {div }}$ is also non-trivial (i.e., $V_{h, k}^{\text {div }} \neq\{0\}$ ).

Corollary 2 There exist a constant $\beta>0$, independent of $h$ and $v$, such that

$$
\inf _{q_{h} \in C_{h, k}^{1} \operatorname{v}_{h} \in\left[V_{h}^{k}\right]^{d}} \frac{\left|b_{h}\left(q_{h}, \mathbf{v}_{h}\right)\right|}{\left\|q_{h}\right\|_{0, \Omega}\left\|\mathbf{v}_{h}\right\|_{1, \Omega}} \geq \beta .
$$

We now may state the main result of this paragraph.

Theorem 1 The discrete problem (9) with $\mathbf{u}_{0, h} \in V_{h, k}^{\text {div }}$ has a unique solution $\left(\mathbf{u}_{h}, p_{h}\right) \in$ $C^{1}\left(0, T ;\left[V_{h}^{k}\right]^{d}\right) \times C^{0}\left(0, T ; Q_{h}^{k}\right)$. 
Proof The result follows from the Cauchy-Lipschitz theorem and Corollary 2. We refer to [9] for the details (see also [10]).

Remark 2 In order to ensure convergence, in the following we shall set $\mathbf{u}_{0, h}=P_{h}^{k} \mathbf{u}_{0} \in$ $V_{h, k}^{\text {div }}$.

\subsection{Coercivity}

The following Lemma provides control (uniform in $v$ ) of the divergence constraint through the stabilization terms.

Lemma 7 (Divergence control) Assume $\left(\mathbf{u}_{h}, p_{h}\right) \in W_{h}^{k}$ be a solution of (9). There exists a constant $C>0$, depending only on the mesh geometry, such that

$$
C\left\|h^{\frac{1}{2}} \nabla \cdot \mathbf{u}_{h}\right\|_{0, \Omega}^{2} \leq \mathbf{J}\left[\mathbf{0} ;\left(\mathbf{u}_{h}, p_{h}\right),\left(\mathbf{u}_{h}, p_{h}\right)\right]+\left\|\mathbf{u}_{h} \cdot \mathbf{n}\right\|_{0, \partial \Omega}^{2} .
$$

Proof By testing (9) with $\mathbf{v}_{h}=\mathbf{0}$ we get

$$
\left(q_{h}, \nabla \cdot \mathbf{u}_{h}\right)-\left\langle q_{h}, \mathbf{u}_{h} \cdot \mathbf{n}\right\rangle_{\partial \Omega}+j\left(p_{h}, q_{h}\right)=0 .
$$

Thus, taking $q_{h}=\pi_{h}^{*}\left(h \nabla \cdot \mathbf{u}_{h}\right)$ yields

$$
\begin{aligned}
& \left\|h^{\frac{1}{2}} \nabla \cdot \mathbf{u}_{h}\right\|_{0, \Omega}^{2}+\left(\nabla \cdot \mathbf{u}_{h}, \pi_{h}^{*}\left(h \nabla \cdot \mathbf{u}_{h}\right)-h \nabla \cdot \mathbf{u}_{h}\right) \\
& \quad=\left\langle\pi_{h}^{*}\left(h \nabla \cdot \mathbf{u}_{h}\right), \mathbf{u}_{h} \cdot \mathbf{n}\right\rangle_{\partial \Omega}-j\left(p_{h}, \pi_{h}^{*}\left(h \nabla \cdot \mathbf{u}_{h}\right)\right) .
\end{aligned}
$$

It follows then, by the quasi-uniformity of the mesh, a Cauchy-Schwarz inequality, a trace inequality and an inverse inequality, that

$$
\begin{aligned}
& \frac{1}{2}\left\|h^{\frac{1}{2}} \nabla \cdot \mathbf{u}_{h}\right\|_{0, \Omega}^{2} \leq \frac{1}{2}\left\|h^{\frac{1}{2}}\left(\pi_{h}^{*}\left(\nabla \cdot \mathbf{u}_{h}\right)-\nabla \cdot \mathbf{u}_{h}\right)\right\|_{0, \Omega}^{2} \\
& \quad+C\left(\left\|\mathbf{u}_{h} \cdot \mathbf{n}\right\|_{0, \partial \Omega}+j^{\frac{1}{2}}\left(p_{h}, p_{h}\right)\right)\left\|h^{\frac{1}{2}} \pi_{h}^{*}\left(\nabla \cdot \mathbf{u}_{h}\right)\right\|_{0, \Omega} .
\end{aligned}
$$

Therefore, using the triangle inequality and the $L^{2}$-stability of the Oswald interpolant, this yields

$$
\begin{aligned}
\frac{1}{2}\left\|h^{\frac{1}{2}} \nabla \cdot \mathbf{u}_{h}\right\|_{0, \Omega}^{2} \leq & C\left\|h^{\frac{1}{2}}\left(\pi_{h}^{*}\left(\nabla \cdot \mathbf{u}_{h}\right)-\nabla \cdot \mathbf{u}_{h}\right)\right\|_{0, \Omega}^{2} \\
& +C\left(\left\|\mathbf{u}_{h} \cdot \mathbf{n}\right\|_{0, \partial \Omega}^{2}+j\left(p_{h}, p_{h}\right)\right) \\
& +C\left(\left\|\mathbf{u}_{h} \cdot \mathbf{n}\right\|_{0, \partial \Omega}+j^{\frac{1}{2}}\left(p_{h}, p_{h}\right)\right)\left\|h^{\frac{1}{2}} \nabla \cdot \mathbf{u}_{h}\right\|_{0, \Omega}
\end{aligned}
$$

We conclude using a Young's inequality and the interpolation result (27).

Using Lemma 7 we may now show that the bilinear form is coercive for the triple norm $\|\cdot\|_{\mathbf{w}_{h}}$. 
Lemma 8 (Coercivity) There exits a constant $C_{\mathrm{A}}>0$, depending only on $\Omega$ and $\gamma_{\nu}$, such that

$$
(\mathbf{A}+\mathbf{J})\left[\mathbf{w}_{h} ;\left(\mathbf{v}_{h}, q_{h}\right),\left(\mathbf{v}_{h}, q_{h}\right)\right] \geq C_{\mathrm{A}}\left\|\left(\mathbf{v}_{h}, q_{h}\right)\right\|_{\mathbf{w}_{h}}^{2},
$$

for all $\left(\mathbf{w}_{h},\left(\mathbf{v}_{h}, q_{h}\right)\right) \in\left[V_{h}^{k}\right]^{d} \times W_{h}^{k}$.

Proof From (9) we have

$$
\begin{aligned}
& (\mathbf{A}+\mathbf{J})\left[\mathbf{w}_{h} ;\left(\mathbf{v}_{h}, q_{h}\right),\left(\mathbf{v}_{h}, q_{h}\right)\right] \geq 2\left\|v^{\frac{1}{2}} \boldsymbol{\varepsilon}\left(\mathbf{v}_{h}\right)\right\|_{0, \Omega}^{2} \\
& \quad+\mathbf{J}\left[\mathbf{w}_{h} ;\left(\mathbf{v}_{h}, q_{h}\right),\left(\mathbf{v}_{h}, q_{h}\right)\right]+\left\|\gamma_{\nu}^{\frac{1}{2}}(v / h)^{\frac{1}{2}} \mathbf{v}_{h}\right\|_{0, \partial \Omega}^{2} \\
& \quad+\left\|\mathbf{v}_{h} \cdot \mathbf{n}\right\|_{0, \partial \Omega}^{2}-\left\langle 4 v \boldsymbol{\varepsilon}\left(\mathbf{v}_{h}\right) \mathbf{n}, \mathbf{v}_{h}\right\rangle_{\partial \Omega},
\end{aligned}
$$

where we used the fact that, after integration by parts,

$$
\left(\mathbf{w}_{h} \cdot \nabla \mathbf{v}_{h}, \mathbf{v}_{h}\right)=\frac{1}{2}\left[\left\langle\mathbf{w}_{h} \cdot \mathbf{n} \mathbf{v}_{h}, \mathbf{v}_{h}\right\rangle_{\partial \Omega}-\left(\nabla \cdot \mathbf{w}_{h} \mathbf{v}_{h}, \mathbf{v}_{h}\right)\right] .
$$

The last term in (38) can be bounded using the Cauchy-Schwarz inequality followed by (25) and the quasi-uniformity of the mesh (7), to obtain

$$
\left|\left\langle 4 v \boldsymbol{\varepsilon}\left(\mathbf{v}_{h}\right) \mathbf{n}, \mathbf{v}_{h}\right\rangle_{\partial \Omega}\right| \leq 8 \frac{C_{\mathrm{T}}}{C_{\mathrm{U} \gamma_{\nu}}}\left\|v^{\frac{1}{2}} \boldsymbol{\varepsilon}\left(\mathbf{v}_{h}\right)\right\|_{0, \Omega}^{2}+\frac{1}{2}\left\|\gamma_{\nu}^{\frac{1}{2}}\left(\frac{v}{h}\right)^{\frac{1}{2}} \mathbf{v}_{h}\right\|_{0, \partial \Omega}^{2}
$$

In what follows we will assume that

$$
\gamma_{v}>4 \frac{C_{\mathrm{T}}}{C_{\mathrm{U}}}>0
$$

and therefore

$$
\lambda\left(\gamma_{\nu}\right) \stackrel{\text { def }}{=} 2-8 \frac{C_{\mathrm{T}}}{C_{\mathrm{U} \gamma_{\nu}}}>0
$$

From (38), we then get

$$
\begin{aligned}
& (\mathbf{A}+\mathbf{J})\left[\mathbf{w}_{h} ;\left(\mathbf{v}_{h}, q_{h}\right),\left(\mathbf{v}_{h}, q_{h}\right)\right] \geq \lambda\left(\gamma_{v}\right)\left\|v^{\frac{1}{2}} \boldsymbol{\varepsilon}\left(\mathbf{v}_{h}\right)\right\|_{0, \Omega}^{2} \\
& +\mathbf{J}\left[\mathbf{w}_{h} ;\left(\mathbf{v}_{h}, q_{h}\right),\left(\mathbf{v}_{h}, q_{h}\right)\right]+\frac{1}{2}\left\|\gamma_{\nu}^{\frac{1}{2}}(v / h)^{\frac{1}{2}} \mathbf{v}_{h}\right\|_{0, \partial \Omega}^{2} \\
& +\left\|\mathbf{v}_{h} \cdot \mathbf{n}\right\|_{0, \partial \Omega}^{2},
\end{aligned}
$$


and consequently

$$
\begin{aligned}
& (\mathbf{A}+\mathbf{J})\left[\mathbf{w}_{h} ;\left(\mathbf{v}_{h}, q_{h}\right),\left(\mathbf{v}_{h}, q_{h}\right)\right] \\
& \geq \min \left\{\lambda\left(\gamma_{v}\right), \frac{\gamma_{v}}{4 h}\right\}\left(\left\|v^{\frac{1}{2}} \boldsymbol{\varepsilon}\left(\mathbf{v}_{h}\right)\right\|_{0, \Omega}^{2}+\left\|v^{\frac{1}{2}} \mathbf{v}_{h}\right\|_{0, \partial \Omega}^{2}\right) \\
& \quad+\mathbf{J}\left[\mathbf{w}_{h} ;\left(\mathbf{v}_{h}, q_{h}\right),\left(\mathbf{v}_{h}, q_{h}\right)\right]+\frac{1}{4}\left\|\gamma_{\nu}^{\frac{1}{2}}(v / h)^{\frac{1}{2}} \mathbf{v}_{h}\right\|_{0, \partial \Omega}^{2} \\
& \quad+\left\|\mathbf{v}_{h} \cdot \mathbf{n}\right\|_{0, \partial \Omega}^{2} .
\end{aligned}
$$

In particular, by choosing (accordingly with (39))

$$
\gamma_{v} \stackrel{\text { def }}{=} \frac{1}{8}+4 \frac{C_{\mathrm{T}}}{C_{\mathrm{U}}},
$$

and since $0<h \leq 1$, one obtains

$$
\lambda\left(\gamma_{\nu}\right)<\frac{\gamma_{v}}{4 h}
$$

We conclude the proof using Korn's inequality and Lemma 7.

\section{Convergence}

We now prove convergence first of the velocities and then of the pressures. Since the problem decomposes into one linear part and one non-linear part it is convenient first to recall a preliminary result regarding the continuity of the Stokes system from [10].

Lemma 9 There exists a constant $C>0$, independent of $v$ and $h$, such that

$$
a_{h}\left(\mathbf{v}, \mathbf{v}_{h}\right)-b_{h}\left(q, \mathbf{v}_{h}\right)+b_{h}\left(q_{h}, \mathbf{v}\right) \leq C\|(\mathbf{v}, q)\|\left\|\left(\mathbf{v}_{h}, q_{h}\right)\right\|_{\mathbf{0}},
$$

for all $(q, \mathbf{v}) \in\left[\left(V_{h}^{k}\right)^{\perp} \times\left(\left[V_{h}^{k}\right]^{d}\right)^{\perp}\right] \cap\left[H^{\frac{1}{2}+\epsilon}\left(\mathcal{T}_{h}\right) \times\left[H^{\frac{3}{2}+\epsilon}\left(\mathcal{T}_{h}\right)\right]^{d}\right]$ and $\left(q_{h}, \mathbf{v}_{h}\right) \in$ $V_{h}^{k} \times\left[V_{h}^{k}\right]^{d}$.

Proof Using Cauchy-Schwarz and the trace inequality (25) and since $0<h$, $v \leq 1$, for the first term one readily obtains

$$
a_{h}\left(\mathbf{v}, \mathbf{v}_{h}\right) \leq C\|(\mathbf{v}, 0) \llbracket\|\left\|\left(\mathbf{v}_{h}, 0\right)\right\|_{\mathbf{0}} .
$$

For the second term we have, using the orthogonality of $q$ (to $V_{h}^{k}$ ) and the interpolation estimate (27),

$$
\begin{aligned}
b_{h}\left(q, \mathbf{v}_{h}\right)= & -\left(q, \nabla \cdot \mathbf{v}_{h}-\pi_{h}^{*}\left(\nabla \cdot \mathbf{v}_{h}\right)\right)+\left\langle q, \mathbf{v}_{h} \cdot \mathbf{n}\right\rangle_{\partial \Omega} \\
\leq & \left\|h^{-\frac{1}{2}} q\right\|_{0, \Omega}\left\|h^{\frac{1}{2}}\left(\nabla \cdot \mathbf{v}_{h}-\pi_{h}^{*}\left(\nabla \cdot \mathbf{v}_{h}\right)\right)\right\|_{0, \Omega} \\
& +\|q\|_{0, \partial \Omega}\left\|\mathbf{v}_{h} \cdot \mathbf{n}\right\|_{0, \partial \Omega} \\
\leq & C\|(0, q)\|\left\|\left(\mathbf{v}_{h}, 0\right)\right\|_{\mathbf{0}} .
\end{aligned}
$$


In a similar fashion, after integration by parts in the third term, one obtains

$$
\begin{aligned}
b_{h}\left(q_{h}, \mathbf{v}\right) & =-\left(q_{h}, \nabla \cdot \mathbf{v}\right)+\left\langle q_{h}, \mathbf{v} \cdot \mathbf{n}\right\rangle_{\partial \Omega} \\
& =\left(\nabla q_{h}, \mathbf{v}\right) \\
& =\left(\nabla q_{h}-\pi_{h}^{*}\left(\nabla q_{h}\right), \mathbf{v}\right) \\
& \leq\left\|h^{\frac{1}{2}}\left(\nabla q_{h}-\pi_{h}^{*}\left(\nabla q_{h}\right)\right)\right\|_{0, \Omega}\left\|h^{-\frac{1}{2}} \mathbf{v}\right\|_{0, \Omega} \\
& \leq C\|(\mathbf{v}, 0)\|\left\|\left(\mathbf{0}, q_{h}\right)\right\|_{\mathbf{0}} .
\end{aligned}
$$

Hence, the proof is complete.

\subsection{Velocity energy norm error estimate}

The following theorem states the main result of this paragraph.

Theorem 2 Let $(\mathbf{u}, p)$ the solution of (1), $\left(\mathbf{u}_{h}, p_{h}\right) \in W_{h}^{k}$ the solution of (9), with $\mathbf{u}_{0, h}=P_{h}^{k} \mathbf{u}_{0}$, and assume that (u, p) has the minimal regularity (4). Then, the following optimal approximation estimates hold

$$
\begin{aligned}
& \left\|\Pi_{h}^{k} \mathbf{u}-\mathbf{u}_{h}\right\|_{L^{\infty}\left(0, T ; L^{2}(\Omega)\right)}^{2} \leq c_{\exp }\left\|\Pi_{h}^{k} \mathbf{u}_{0}-\mathbf{u}_{0, h}\right\|_{0, \Omega}^{2} \\
& +c_{\exp } \int_{0}^{T}\left(c_{1}\left\|\left(\mathbf{u}-\Pi_{h}^{k} \mathbf{u}, 0\right)\right\|^{2}+c_{2} \mathbf{J}\left[\mathbf{0} ;\left(\Pi_{h}^{k} \mathbf{u}, \Pi_{h}^{k} p\right),\left(\Pi_{h}^{k} \mathbf{u}, \Pi_{h}^{k} p\right)\right]\right) \mathrm{d} t,
\end{aligned}
$$

and

$$
\begin{aligned}
& \int_{0}^{T}\left\|\left(\Pi_{h}^{k} \mathbf{u}-\mathbf{u}_{h}, \Pi_{h}^{k} p-p_{h}\right)\right\|_{\mathbf{u}_{h}}^{2} \mathrm{~d} t \leq c_{\exp }\left\|\Pi_{h}^{k} \mathbf{u}_{0}-\mathbf{u}_{0, h}\right\|_{0, \Omega}^{2} \\
& \quad+c_{\exp } \int_{0}^{T}\left(c_{1}\left\|\left(\mathbf{u}-\Pi_{h}^{k} \mathbf{u}, 0\right)\right\|^{2}+c_{2} \mathbf{J}\left[\mathbf{0} ;\left(\Pi_{h}^{k} \mathbf{u}, \Pi_{h}^{k} p\right),\left(\Pi_{h}^{k} \mathbf{u}, \Pi_{h}^{k} p\right)\right]\right) \mathrm{d} t,
\end{aligned}
$$

with

$$
\begin{aligned}
c_{\text {exp }} & \stackrel{\text { def }}{=} e^{C T\left(h\left\|\mathbf{u}_{L^{\infty}\left(0, T ; W^{1, \infty}(\Omega)\right)}^{2}+\right\| \mathbf{u}_{L^{\infty}\left(0, T ; W^{1, \infty}(\Omega)\right)}\right)}, \\
c_{1} & \stackrel{\text { def }}{=} C\left(1+h^{\frac{1}{2}}\|\mathbf{u}\|_{L^{\infty}\left(0, T ; W^{1, \infty}(\Omega)\right)}\right), \\
c_{2} & \stackrel{\text { def }}{=} C\left(1+\|\mathbf{u}\|_{L^{\infty}\left(0, T ; L^{\infty}(\Omega)\right)}^{2}+h^{2}\|\mathbf{u}\|_{L^{\infty}\left(0, T ; W^{1, \infty}(\Omega)\right)}^{2}\right),
\end{aligned}
$$

with $C>0$ a positive constant independent of $v$ and $h$. 
We stress that the constants in the above theorem have no explicit dependence on $v$. Before proving the main convergence theorem we state two immediate consequences in the form of corollaries.

Corollary 3 Under the hypothesis of the previous theorem, assuming that the exact solution $(\mathbf{u}, p)$ has the regularity given in (5) and that $v<h$, the following error estimates hold

$$
\left\|\mathbf{u}-\mathbf{u}_{h}\right\|_{L^{\infty}\left(0, T ; L^{2}(\Omega)\right)}^{2} \leq C_{\mathbf{u}} h^{2 r_{\mathbf{u}}-1}+C_{p} h^{2 r_{p}-1}
$$

and

$$
\int_{0}^{T}\left\|\left(\mathbf{u}-\mathbf{u}_{h}, p-p_{h}\right)\right\|_{\mathbf{u}_{h}}^{2} \mathrm{~d} t \leq \widetilde{C}_{\mathbf{u}} h^{2 r_{\mathbf{u}}-1}+C_{p} h^{2 r_{p}-1},
$$

with $r_{\mathbf{u}} \stackrel{\text { def }}{=} \min \{r, k+1\}, r_{p} \stackrel{\text { def }}{=} \min \{s, k+1\}$ and

$$
\begin{aligned}
& C_{\mathbf{u}} \stackrel{\text { def }}{=} C\|\mathbf{u}\|_{L^{\infty}\left(0, T ; H^{r} \mathbf{u}(\Omega)\right)}^{2}+\widetilde{C}_{\mathbf{u}}, \\
& \widetilde{C}_{\mathbf{u}} \stackrel{\text { def }}{=} C\left(c_{\exp }, c_{1}, c_{2}\right)\left(\|\mathbf{u}\|_{L^{2}\left(0, T ; H^{r}(\Omega)\right)}^{2}+\left\|\mathbf{u}_{0}\right\|_{r_{\mathbf{u}}, \Omega}^{2}\right), \\
& C_{p} \stackrel{\text { def }}{=} C\left(c_{\exp }, c_{1}, c_{2}\right)\|p\|_{L^{2}\left(0, T ; H^{r}(\Omega)\right)}^{2},
\end{aligned}
$$

and $C, C\left(c_{\exp }, c_{1}, c_{2}\right)$ two positive constants independent of $v$ and $h$.

Proof Immediate by a triangle inequality, the result of Theorem 2 and approximation (Lemmas 4 and 5).

Corollary 4 Under the hypothesis of the previous corollary, the following error estimate holds:

$$
\begin{aligned}
\left\|\mathbf{u}-\mathbf{u}_{h}\right\|_{L^{\infty}\left(0, T ; L^{\infty}(\Omega)\right)}^{2} \leq & C h^{2}\|\mathbf{u}\|_{L^{\infty}\left(0, T ; W^{1, \infty}(\Omega)\right)}^{2} \\
& +h^{-d}\left(C_{\mathbf{u}} h^{2 r_{\mathbf{u}}-1}+C_{p} h^{2 r_{p}-1}\right),
\end{aligned}
$$

with $C>0$ a positive constant independent of $v$ and $h$. In particular, there holds $\mathbf{u}_{h} \in L^{\infty}(\Omega \times(0, T))$.

Proof Immediate using approximation (22), an inverse inequality and (40). 


\subsection{Proof of theorem 2}

In the following, $\epsilon_{i}>0$ for $i=1,2, \ldots$, represents a free positive constant to be fixed later on. We denote the discrete and projection errors as

$$
\begin{gathered}
\boldsymbol{\theta}_{h} \stackrel{\text { def }}{=} \Pi_{h}^{k} \mathbf{u}-\mathbf{u}_{h}, \quad \boldsymbol{\theta}^{\pi} \stackrel{\text { def }}{=} \mathbf{u}-\Pi_{h}^{k} \mathbf{u}, \\
y_{h} \stackrel{\text { def }}{=} \Pi_{h}^{k} p-p_{h}, \quad y^{\pi}=p-\Pi_{h}^{k} p,
\end{gathered}
$$

which gives

$$
\boldsymbol{\theta}_{h}=\mathbf{u}-\mathbf{u}_{h}-\boldsymbol{\theta}^{\pi}, \quad y_{h}=p-p_{h}-y^{\pi} .
$$

Note that, since $\mathbf{u} \in H^{1}\left(0, T ; L^{2}(\Omega)\right)$, we may deduce that $\boldsymbol{\theta}_{h} \in$ $H^{1}\left(0, T ; L^{2}(\Omega)\right)$. Using coercivity (Lemma 8$)$ we then get

$$
\begin{aligned}
& \frac{1}{2} \frac{\mathrm{d}}{\mathrm{d} t}\left\|\boldsymbol{\theta}_{h}\right\|_{0,2}^{2}+C_{\mathrm{A}}\left\|\left(\boldsymbol{\theta}_{h}, y_{h}\right)\right\|_{\mathbf{u}_{h}}^{2} \\
& \quad \leq\left(\partial_{t} \boldsymbol{\theta}_{h}, \boldsymbol{\theta}_{h}\right)+(\mathbf{A}+\mathbf{J})\left[\mathbf{u}_{h} ;\left(\boldsymbol{\theta}_{h}, y_{h}\right),\left(\boldsymbol{\theta}_{h}, y_{h}\right)\right] .
\end{aligned}
$$

Hence, from (42)-(43) and the tri-linearity of $\mathbf{A}$ and $\mathbf{J}$, we have

$$
\begin{aligned}
\frac{1}{2} \frac{\mathrm{d}}{\mathrm{d} t}\left\|\boldsymbol{\theta}_{h}\right\|_{0,2}^{2}+C_{\mathrm{A}}\left\|\left(\boldsymbol{\theta}_{h}, y_{h}\right)\right\|_{\mathbf{u}_{h} \leq}^{2} \leq & \left(\partial_{t}\left(\mathbf{u}-\mathbf{u}_{h}\right), \boldsymbol{\theta}_{h}\right)-\left(\partial_{t} \boldsymbol{\theta}^{\pi}, \boldsymbol{\theta}_{h}\right) \\
& +(\mathbf{A}+\mathbf{J})\left[\mathbf{u}_{h} ;\left(\Pi_{h}^{k} \mathbf{u}, \Pi_{h}^{k} p\right)\left(\boldsymbol{\theta}_{h}, y_{h}\right)\right] \\
& -(\mathbf{A}+\mathbf{J})\left[\mathbf{u}_{h} ;\left(\mathbf{u}_{h}, p_{h}\right),\left(\boldsymbol{\theta}_{h}, y_{h}\right)\right] .
\end{aligned}
$$

Thus, by testing the approximate Galerkin orthogonality (Lemma 1) with $\left(\mathbf{v}_{h}, q_{h}\right)=$ $\left(\boldsymbol{\theta}_{h}, y_{h}\right)$ and since since $\left(\partial_{t} \boldsymbol{\theta}^{\pi}, \boldsymbol{\theta}_{h}\right)=0$, we obtain

$$
\begin{aligned}
\frac{1}{2} \frac{\mathrm{d}}{\mathrm{d} t}\left\|\boldsymbol{\theta}_{h}\right\|_{0,2}^{2}+C_{\mathrm{A}}\left\|\left(\boldsymbol{\theta}_{h}, y_{h}\right)\right\|_{\mathbf{u}_{h}}^{2} \leq & (\mathbf{A}+\mathbf{J})\left[\mathbf{u}_{h} ;\left(\Pi_{h}^{k} \mathbf{u}, \Pi_{h}^{k} p\right)\left(\boldsymbol{\theta}_{h}, y_{h}\right)\right] \\
& -\mathbf{A}\left[\mathbf{u} ;(\mathbf{u}, p),\left(\boldsymbol{\theta}_{h}, y_{h}\right)\right]
\end{aligned}
$$

Writing out all the terms of $\mathbf{A}$ and $\mathbf{J}$, as given in (10), we get

$$
\begin{aligned}
& \frac{1}{2} \frac{\mathrm{d}}{\mathrm{d} t}\left\|\boldsymbol{\theta}_{h}\right\|_{0,2}^{2}+C_{\mathrm{A}}\left\|\left(\boldsymbol{\theta}_{h}, y_{h}\right)\right\|_{\mathbf{u}_{h}}^{2} \leq-a_{h}\left(\boldsymbol{\theta}^{\pi}, \boldsymbol{\theta}_{h}\right)-b_{h}\left(y^{\pi}, \boldsymbol{\theta}_{h}\right)+b_{h}\left(y_{h}, \boldsymbol{\theta}^{\pi}\right) \\
& \quad+\gamma j\left(\Pi_{h}^{k} \mathbf{u}, \boldsymbol{\theta}_{h}\right)+j\left(\Pi_{h}^{k} p, y_{h}\right)+c_{h}\left(\mathbf{u}_{h} ; \Pi_{h}^{k} \mathbf{u}, \boldsymbol{\theta}_{h}\right)-c\left(\mathbf{u} ; \mathbf{u}, \boldsymbol{\theta}_{h}\right) \\
& \quad+j_{\mathbf{u}_{h}}\left(\Pi_{h}^{k} \mathbf{u}, \boldsymbol{\theta}_{h}\right) .
\end{aligned}
$$


Now we may use the continuity of the Stokes system (Lemma 9) to obtain,

$$
\begin{aligned}
& \frac{1}{2} \frac{\mathrm{d}}{\mathrm{d} t}\left\|\boldsymbol{\theta}_{h}\right\|_{0,2}^{2}+C_{\mathrm{A}}\left\|\left(\boldsymbol{\theta}_{h}, y_{h}\right)\right\|_{\mathbf{u}_{h}}^{2} \\
& \leq C_{1}\left(\|\left(\boldsymbol{\theta}^{\pi}, y^{\pi}\right) \rrbracket+\mathbf{J}^{\frac{1}{2}}\left[\mathbf{0} ;\left(\Pi_{h}^{k} \mathbf{u}, \Pi_{h}^{k} p\right),\left(\Pi_{h}^{k} \mathbf{u}, \Pi_{h}^{k} p\right)\right]\right)\left\|\left(\boldsymbol{\theta}_{h}, y_{h}\right)\right\|_{\mathbf{0}} \\
& +\left(\mathbf{u}_{h} \cdot \nabla \Pi_{h}^{k} \mathbf{u}, \boldsymbol{\theta}_{h}\right)+\frac{1}{2}\left(\nabla \cdot \mathbf{u}_{h} \Pi_{h}^{k} \mathbf{u}, \boldsymbol{\theta}_{h}\right) \\
& \quad-\frac{1}{2}\left\langle\mathbf{u}_{h} \cdot \mathbf{n} \Pi_{h}^{k} \mathbf{u}, \boldsymbol{\theta}_{h}\right\rangle_{\partial \Omega}-\left(\mathbf{u} \cdot \nabla \mathbf{u}, \boldsymbol{\theta}_{h}\right) \\
& \quad+\sum_{K \in \mathcal{T}_{h}} \int_{h_{K}} h_{K}^{2}\left|\mathcal{I}_{h}^{1} \mathbf{u}_{h}\right|^{2} \llbracket \nabla \Pi_{h}^{k} \mathbf{u} \rrbracket: \llbracket \nabla \boldsymbol{\theta}_{h} \rrbracket \mathrm{d} \mathbf{s}
\end{aligned}
$$

Using (43), this leads to

$$
\begin{aligned}
& \frac{1}{2} \frac{\mathrm{d}}{\mathrm{d} t}\left\|\boldsymbol{\theta}_{h}\right\|_{0,2}^{2}+C_{\mathrm{A}}\left\|\left(\boldsymbol{\theta}_{h}, y_{h}\right)\right\|_{\mathbf{u}_{h}}^{2} \leq \\
& C_{1}\left(\|\left(\boldsymbol{\theta}^{\pi}, y^{\pi}\right) \rrbracket+\mathbf{J}^{\frac{1}{2}}\left[\mathbf{0} ;\left(\Pi_{h}^{k} \mathbf{u}, \Pi_{h}^{k} p\right),\left(\Pi_{h}^{k} \mathbf{u}, \Pi_{h}^{k} p\right)\right]\right)\left\|\left(\boldsymbol{\theta}_{h}, y_{h}\right)\right\|_{\mathbf{0}} \\
& \quad-\left(\mathbf{u}_{h} \cdot \nabla \boldsymbol{\theta}^{\pi}, \boldsymbol{\theta}_{h}\right)-\frac{1}{2}\left(\nabla \cdot \mathbf{u}_{h} \boldsymbol{\theta}^{\pi}, \boldsymbol{\theta}_{h}\right) \\
& \quad+\frac{1}{2}\left\langle\mathbf{u}_{h} \cdot \mathbf{n} \boldsymbol{\theta}^{\pi}, \boldsymbol{\theta}_{h}\right\rangle_{\partial \Omega}+\left(\left(\mathbf{u}_{h}-\mathbf{u}\right) \cdot \nabla \mathbf{u}, \boldsymbol{\theta}_{h}\right) \\
& \quad+\frac{1}{2}\left(\nabla \cdot \mathbf{u}_{h} \mathbf{u}, \boldsymbol{\theta}_{h}\right)+\sum_{K \in \mathcal{T}_{h}} \int_{\partial} h_{K}^{2}\left|\mathcal{I}_{h}^{1} \mathbf{u}_{h} \cdot \mathbf{n}\right|^{2} \llbracket \nabla \Pi_{h}^{k} \mathbf{u} \rrbracket: \llbracket \nabla \boldsymbol{\theta}_{h} \rrbracket \mathrm{d} \boldsymbol{s},
\end{aligned}
$$

which, after integration by parts in the convective term, gives

$$
\begin{aligned}
& \frac{1}{2} \frac{\mathrm{d}}{\mathrm{d} t}\left\|\boldsymbol{\theta}_{h}\right\|_{0,2}^{2}+C_{\mathrm{A}}\left\|\left(\boldsymbol{\theta}_{h}, y_{h}\right)\right\|_{\mathbf{u}_{h}}^{2} \leq \frac{C_{1} \epsilon_{1}}{2}\left\|\left(\boldsymbol{\theta}_{h}, y_{h}\right)\right\|_{\mathbf{0}}^{2} \\
& +\frac{C_{1}}{2 \epsilon_{1}}\left(\left\|\left(\boldsymbol{\theta}^{\pi}, y^{\pi}\right) \llbracket\right\|^{2}+\mathbf{J}\left[\mathbf{0},\left(\Pi_{h}^{k} \mathbf{u}, \Pi_{h}^{k} p\right),\left(\Pi_{h}^{k} \mathbf{u}, \Pi_{h}^{k} p\right)\right]\right) \\
& +\underbrace{\left(\boldsymbol{\theta}^{\pi}, \mathbf{u}_{h} \cdot \nabla \boldsymbol{\theta}_{h}\right)}_{T_{1}}+\underbrace{\frac{1}{2}\left(\nabla \cdot \mathbf{u}_{h} \boldsymbol{\theta}^{\pi}, \boldsymbol{\theta}_{h}\right)}_{T_{2}}-\underbrace{\frac{1}{2}\left\langle\mathbf{u}_{h} \cdot \mathbf{n} \boldsymbol{\theta}^{\pi}, \boldsymbol{\theta}_{h}\right\rangle_{\partial \Omega}}_{T_{3}} \\
& +\underbrace{\left(\left(\mathbf{u}_{h}-\mathbf{u}\right) \cdot \nabla \mathbf{u}, \boldsymbol{\theta}_{h}\right)}_{T_{4}}+\underbrace{\frac{1}{2}\left(\nabla \cdot \mathbf{u}_{h} \mathbf{u}, \boldsymbol{\theta}_{h}\right)}_{T_{5}} \\
& +\underbrace{\sum_{K \in \mathcal{T}_{h} K} \int_{T_{K}} h_{K}^{2}\left|\mathcal{I}_{h}^{1} \mathbf{u}_{h} \cdot \mathbf{n}\right|^{2} \llbracket \nabla \Pi_{h}^{k} \mathbf{u} \rrbracket: \llbracket \nabla \boldsymbol{\theta}_{h} \rrbracket \mathrm{d} \boldsymbol{s}}_{T_{6}} .
\end{aligned}
$$


In the next paragraphs we analyze the terms $T_{i}, i=1,2, \ldots, 6$. Using the orthogonality of the $L^{2}$-projection, approximation and Lemma 3, we have

$$
\begin{aligned}
T_{1}= & \underbrace{\left(\boldsymbol{\theta}^{\pi},\left(\mathbf{u}_{h}-\mathcal{I}_{h}^{1} \mathbf{u}_{h}\right) \cdot \nabla \boldsymbol{\theta}_{h}\right)}_{T_{1,1}} \\
& +\underbrace{\left(\boldsymbol{\theta}^{\pi}, \mathcal{I}_{h}^{1} \mathbf{u}_{h} \cdot \nabla \boldsymbol{\theta}_{h}-\pi_{h}^{*}\left(\mathcal{I}_{h}^{1} \mathbf{u}_{h} \cdot \nabla \boldsymbol{\theta}_{h}\right)\right)}_{T_{2,2}} .
\end{aligned}
$$

In the first term, we use the local interpolation property of the $\mathbb{P}_{1}$-interpolant followed by an inverse inequality showing that

$$
\begin{aligned}
\left\|\mathbf{u}_{h}-\mathcal{I}_{h}^{1} \mathbf{u}_{h}\right\|_{0, K} & \leq C_{2} h_{K}^{2}\left|\mathbf{u}_{h}\right|_{2, K} \\
& \leq C_{3}\left\|\mathbf{u}_{h}-\mathcal{I}_{h}^{1} \mathbf{u}\right\|_{0, K} .
\end{aligned}
$$

Using this inequality, for the first term of $T_{1,1}$ we have

$$
\begin{aligned}
T_{1,1} & \leq C_{4} \sum_{K \in \mathcal{T}_{h}}\left\|\mathbf{u}_{h}-\mathcal{I}_{h}^{1} \mathbf{u}_{h}\right\|_{0, K}\left\|\left[\nabla \boldsymbol{\theta}_{h}\right]^{\mathrm{T}} \boldsymbol{\theta}^{\pi}\right\|_{0, K} \\
& \leq C_{5} \sum_{K \in \mathcal{T}_{h}}\left\|\mathbf{u}_{h}-\mathcal{I}_{h}^{1} \mathbf{u}\right\|_{0, K}\left\|\left[\nabla \boldsymbol{\theta}_{h}\right]^{\mathrm{T}} \boldsymbol{\theta}^{\pi}\right\|_{0, K} .
\end{aligned}
$$

We now use the decomposition (43), to obtain

$$
T_{1,1} \leq C_{5} \sum_{K \in \mathcal{T}_{h}}\left(\left\|\boldsymbol{\theta}_{h}\right\|_{0, K}+\left\|\Pi_{h}^{k} \mathbf{u}-\mathcal{I}_{h}^{1} \mathbf{u}\right\|_{0, K}\right)\left\|\left[\nabla \boldsymbol{\theta}_{h}\right]^{\mathrm{T}} \boldsymbol{\theta}^{\pi}\right\|_{0, K}
$$

Thus, using inverse inequalities (16) and the $L^{\infty}$-stability of $\Pi_{h}^{k}$ (22), one gets

$$
\begin{aligned}
T_{1,1} \leq & C_{6} h^{-1} \sum_{K \in \mathcal{T}_{h}}\left\|\boldsymbol{\theta}^{\pi}\right\|_{0, \infty, K}\left\|\boldsymbol{\theta}_{h}\right\|_{0, K}^{2} \\
& +C_{7} \sum_{K \in \mathcal{T}_{h}}\left\|\Pi_{h}^{k} \mathbf{u}-\mathcal{I}_{h}^{1} \mathbf{u}\right\|_{0, \infty, K}\left\|\nabla \boldsymbol{\theta}_{h}\right\|_{0, K}\left\|\boldsymbol{\theta}^{\pi}\right\|_{0, K} \\
\leq & C_{8} h^{-1}\left\|\boldsymbol{\theta}^{\pi}\right\|_{0, \infty, \Omega}\left\|\boldsymbol{\theta}_{h}\right\|_{0, \Omega}^{2} \\
& +C_{9} h^{-\frac{1}{2}}\left\|\Pi_{h}^{k} \mathbf{u}-\mathcal{I}_{h}^{1} \mathbf{u}\right\|_{0, \infty, \Omega} \sum_{K \in \mathcal{T}_{h}}\left\|\boldsymbol{\theta}_{h}\right\|_{0, K}\left\|h^{-\frac{1}{2}} \boldsymbol{\theta}^{\pi}\right\|_{0, K} \\
\leq & C_{10} h^{-1}\left\|\boldsymbol{\theta}^{\pi}\right\|_{0, \infty, \Omega}\left\|\boldsymbol{\theta}_{h}\right\|_{0, \Omega}^{2} \\
& +C_{11} h^{-\frac{1}{2}}\left\|\mathbf{u}-\mathcal{I}_{h}^{1} \mathbf{u}\right\|_{0, \infty, \Omega}\left(\left\|\boldsymbol{\theta}_{h}\right\|_{0, \Omega}^{2}+\left\|h^{-\frac{1}{2}} \boldsymbol{\theta}^{\pi}\right\|_{0, \Omega}^{2}\right),
\end{aligned}
$$


which, in combination with (20) and approximation, leads to

$$
\begin{aligned}
T_{1,1} & \leq C_{12}\|\nabla \mathbf{u}\|_{0, \infty, \Omega}\left(\left\|\boldsymbol{\theta}_{h}\right\|_{0, \Omega}^{2}+h^{\frac{1}{2}}\left\|h^{-\frac{1}{2}} \boldsymbol{\theta}^{\pi}\right\|_{0, \Omega}^{2}\right) \\
& \leq C_{13}\|\nabla \mathbf{u}\|_{0, \infty, \Omega}\left(\left\|\boldsymbol{\theta}_{h}\right\|_{0, \Omega}^{2}+h^{\frac{1}{2}}\left\|\left(\boldsymbol{\theta}^{\pi}, 0\right)\right\|^{2}\right) .
\end{aligned}
$$

Finally, using Cauchy-Schwarz inequality and (3), we obtain

$$
\begin{aligned}
T_{1,2} & \leq\left\|h^{-\frac{1}{2}} \boldsymbol{\theta}^{\pi}\right\|_{0, \Omega}\left\|h^{\frac{1}{2}}\left(\mathcal{I}_{h}^{1} \mathbf{u}_{h} \cdot \nabla \boldsymbol{\theta}_{h}-\pi_{h}^{*}\left(\mathcal{I}_{h}^{1} \mathbf{u}_{h} \cdot \nabla \boldsymbol{\theta}_{h}\right)\right)\right\|_{0, \Omega} \\
& \leq \frac{1}{2 \epsilon_{2}}\left\|h^{-\frac{1}{2}} \boldsymbol{\theta}^{\pi}\right\|_{0, \Omega}^{2}+\frac{\epsilon_{2} \gamma_{1}}{2} j_{\mathbf{u}_{h}}\left(\boldsymbol{\theta}_{h}, \boldsymbol{\theta}_{h}\right), \\
& \leq \frac{1}{2 \epsilon_{2}}\left\|\left(\boldsymbol{\theta}^{\pi}, 0\right)\right\|^{2}+\frac{\epsilon_{2} \gamma_{1}}{2}\left\|\left(\boldsymbol{\theta}_{h}, 0\right)\right\|_{\mathbf{0}}^{2} .
\end{aligned}
$$

For the second term, we use approximation and that the divergence is included in the triple norm,

$$
\begin{aligned}
T_{2}= & \left.\frac{1}{2}\left(\boldsymbol{\theta}^{\pi},\left(\nabla \cdot \boldsymbol{\theta}_{h}+\nabla \cdot \boldsymbol{\theta}^{\pi}\right) \boldsymbol{\theta}_{h}\right)\right) \\
\leq & \sum_{K \in \mathcal{T}_{h}} h_{K}^{-\frac{1}{2}}\left\|\boldsymbol{\theta}^{\pi}\right\|_{0, \infty, K}\left(\left\|h_{K}^{\frac{1}{2}} \nabla \cdot \boldsymbol{\theta}_{h}\right\|_{0, K}+\left\|h_{K}^{\frac{1}{2}} \nabla \cdot \boldsymbol{\theta}^{\pi}\right\|_{0, K}\right)\left\|\boldsymbol{\theta}_{h}\right\|_{0, K} \\
\leq & C_{14} \epsilon_{3} h^{\frac{1}{2}}\|\nabla \mathbf{u}\|_{0, \infty, \Omega}\left(\left\|h^{\frac{1}{2}} \nabla \cdot \boldsymbol{\theta}_{h}\right\|_{0, \Omega}^{2}+\left\|h^{\frac{1}{2}} \nabla \cdot \boldsymbol{\theta}^{\pi}\right\|_{0, \Omega}^{2}\right) \\
& +\frac{C_{14}}{\epsilon_{3}} h^{\frac{1}{2}}\|\nabla \mathbf{u}\|_{0, \infty, \Omega}\left\|\boldsymbol{\theta}_{h}\right\|_{0, \Omega}^{2} \\
\leq & C_{15} \epsilon_{3}\|\nabla \mathbf{u}\|_{0, \infty, \Omega}\left(\left\|\left(\boldsymbol{\theta}_{h}, 0\right)\right\|_{\mathbf{0}}^{2}+h^{\frac{1}{2}}\left\|\left(\boldsymbol{\theta}^{\pi}, 0\right) \llbracket\right\|^{2}\right) \\
& +\frac{C_{15}}{\epsilon_{3}} h^{\frac{1}{2}}\|\nabla \mathbf{u}\|_{0, \infty, \Omega}\left\|\boldsymbol{\theta}_{h}\right\|_{0, \Omega}^{2} .
\end{aligned}
$$

In this last inequality we used the fact that $0<h \leq 1$.

For the third term, using (43), we have

$$
\begin{aligned}
T_{3} & =\frac{1}{2}\left\langle\left(\mathbf{u}_{h}-\mathbf{u}\right) \cdot \mathbf{n} \boldsymbol{\theta}^{\pi}, \boldsymbol{\theta}_{h}\right\rangle_{\partial \Omega} \\
& =-\frac{1}{2}\left\langle\boldsymbol{\theta}^{\pi} \cdot \mathbf{n} \boldsymbol{\theta}^{\pi}, \boldsymbol{\theta}_{h}\right\rangle_{\partial \Omega}-\frac{1}{2}\left\langle\boldsymbol{\theta}_{h} \cdot \mathbf{n} \boldsymbol{\theta}^{\pi}, \boldsymbol{\theta}_{h}\right\rangle_{\partial \Omega} \\
& \leq \frac{1}{2}\left\|\boldsymbol{\theta}^{\pi}\right\|_{0, \infty, \Omega}\left\|\boldsymbol{\theta}_{h}\right\|_{0, \partial \Omega}\left(\left\|\boldsymbol{\theta}^{\pi} \cdot \mathbf{n}\right\|_{0, \partial \Omega}+\left\|\boldsymbol{\theta}_{h} \cdot \mathbf{n}\right\|_{0, \partial \Omega}\right) .
\end{aligned}
$$

Therefore, using approximation and (25), we conclude that

$$
\begin{aligned}
T_{3} & \leq C_{16} h^{\frac{1}{2}}\|\nabla \mathbf{u}\|_{0, \infty, \Omega}\left\|\boldsymbol{\theta}_{h}\right\|_{0, \Omega}\left(\left\|\left(\boldsymbol{\theta}^{\pi}, 0\right) \rrbracket+\right\|\left(\boldsymbol{\theta}_{h}, 0\right) \|_{\mathbf{0}}\right) \\
& \leq C_{17} \frac{h}{\epsilon_{4}}\|\nabla \mathbf{u}\|_{0, \infty, \Omega}^{2}\left\|\boldsymbol{\theta}_{h}\right\|_{0, \Omega}^{2}+\epsilon_{4} C_{17}\left(\left\|\left(\boldsymbol{\theta}^{\pi}, 0\right) \llbracket\right\|^{2}+\left\|\left(\boldsymbol{\theta}_{h}, 0\right)\right\|_{\mathbf{0}}^{2}\right) .
\end{aligned}
$$


Using again (43) and approximation, for the fourth term we obtain

$$
\begin{aligned}
T_{4} & =-\left(\left(\boldsymbol{\theta}^{\pi}+\boldsymbol{\theta}_{h}\right) \cdot \nabla \mathbf{u}, \boldsymbol{\theta}_{h}\right) \\
& \leq\|\nabla \mathbf{u}\|_{0, \infty, \Omega}\left(\left\|\boldsymbol{\theta}^{\pi}\right\|_{0, \Omega}+\left\|\boldsymbol{\theta}_{h}\right\|_{0, \Omega}\right)\left\|\boldsymbol{\theta}_{h}\right\|_{0, \Omega} \\
& \leq \frac{1}{2}\|\nabla \mathbf{u}\|_{0, \infty, \Omega}\left(h^{\frac{1}{2}}\left\|h^{-\frac{1}{2}} \boldsymbol{\theta}^{\pi}\right\|_{0, \Omega}^{2}+3\left\|\boldsymbol{\theta}_{h}\right\|_{0, \Omega}^{2}\right) \\
& \leq \frac{1}{2}\|\nabla \mathbf{u}\|_{0, \infty, \Omega}\left(h^{\frac{1}{2}}\left\|\left(\boldsymbol{\theta}^{\pi}, 0\right)\right\|^{2}+3\left\|\boldsymbol{\theta}_{h}\right\|_{0, \Omega}^{2}\right) .
\end{aligned}
$$

By testing (9) with $\mathbf{v}_{h}=\mathbf{0}$ and $q_{h}=\Pi_{h}^{k}\left(\mathbf{u} \cdot \boldsymbol{\theta}_{h}\right)$, it follows that

$$
\begin{aligned}
& \left(\nabla \cdot \mathbf{u}_{h}, \Pi_{h}^{k}\left(\mathbf{u} \cdot \boldsymbol{\theta}_{h}\right)\right)-\left\langle\mathbf{u}_{h} \cdot \mathbf{n}, \Pi_{h}^{k}\left(\mathbf{u} \cdot \boldsymbol{\theta}_{h}\right)\right\rangle_{\partial \Omega} \\
& \quad+j\left(p_{h}, \Pi_{h}^{k}\left(\mathbf{u} \cdot \boldsymbol{\theta}_{h}\right)\right)=0 .
\end{aligned}
$$

Thus, inserting this expression into $T_{5}$, one gets

$$
\begin{aligned}
T_{5}= & \frac{1}{2}\left(\nabla \cdot \mathbf{u}_{h}, \mathbf{u} \cdot \boldsymbol{\theta}_{h}\right) \\
= & \frac{1}{2}\left(\nabla \cdot \mathbf{u}_{h},\left(\mathbf{u} \cdot \boldsymbol{\theta}_{h}-\Pi_{h}^{k}\left(\mathbf{u} \cdot \boldsymbol{\theta}_{h}\right)\right)+\frac{1}{2}\left\langle\mathbf{u}_{h} \cdot \mathbf{n}, \Pi_{h}^{k}\left(\mathbf{u} \cdot \boldsymbol{\theta}_{h}\right)\right\rangle_{\partial \Omega}\right. \\
& -\frac{1}{2} j\left(p_{h}, \Pi_{h}^{k}\left(\mathbf{u} \cdot \boldsymbol{\theta}_{h}\right)\right),
\end{aligned}
$$

which, from (43) and the fact that $\mathbf{u}=\mathbf{0}$ on $\partial \Omega$, leads to

$$
\begin{aligned}
T_{5}= & -\underbrace{\frac{1}{2}\left(\nabla \cdot \boldsymbol{\theta}^{\pi}, \mathbf{u} \cdot \boldsymbol{\theta}_{h}-\Pi_{h}^{k}\left(\mathbf{u} \cdot \boldsymbol{\theta}_{h}\right)\right)}_{T_{5,1}} \\
& -\underbrace{\frac{1}{2}\left(\nabla \cdot \boldsymbol{\theta}_{h}, \mathbf{u} \cdot \boldsymbol{\theta}_{h}-\Pi_{h}^{k}\left(\mathbf{u} \cdot \boldsymbol{\theta}_{h}\right)\right)}_{T_{5,2}} \\
& -\underbrace{\frac{1}{2}\left\langle\left(\boldsymbol{\theta}^{\pi}+\boldsymbol{\theta}_{h}\right) \cdot \mathbf{n}, \Pi_{h}^{k}\left(\mathbf{u} \cdot \boldsymbol{\theta}_{h}\right)-\mathbf{u} \cdot \boldsymbol{\theta}_{h}\right\rangle_{\partial \Omega}}_{T_{5,3}} \\
& -\underbrace{\frac{1}{2} j\left(p_{h}, \Pi_{h}^{k}\left(\mathbf{u} \cdot \boldsymbol{\theta}_{h}\right)\right)}_{T_{5,4}} .
\end{aligned}
$$


Each of these terms are treated separately. Using approximation and Corollary 1 we have

$$
\begin{aligned}
T_{5,1} & \leq \frac{1}{2}\left\|h^{\frac{1}{2}} \nabla \cdot \boldsymbol{\theta}^{\pi}\right\|_{0, \Omega}^{2}+\frac{1}{2}\left\|h^{-\frac{1}{2}}\left(\mathbf{u} \cdot \boldsymbol{\theta}_{h}-\Pi_{h}^{k}\left(\mathbf{u} \cdot \boldsymbol{\theta}_{h}\right)\right)\right\|_{0, \Omega}^{2} \\
& \leq C_{18}\left(\left\|\left(\boldsymbol{\theta}^{\pi}, 0\right)\right\|^{2}+h\|\mathbf{u}\|_{1, \infty, \Omega}^{2}\left\|\boldsymbol{\theta}_{h}\right\|_{0, \Omega}^{2}\right) .
\end{aligned}
$$

Using again Corollary 1, it follows that

$$
\begin{aligned}
T_{5,2} & \leq \frac{1}{2}\left\|h^{\frac{1}{2}} \nabla \cdot \boldsymbol{\theta}_{h}\right\|_{0, \Omega}\left\|h^{-\frac{1}{2}}\left(\mathbf{u} \cdot \boldsymbol{\theta}_{h}-\Pi_{h}^{k}\left(\mathbf{u} \cdot \boldsymbol{\theta}_{h}\right)\right)\right\|_{0, \Omega} \\
& \leq C_{19} \epsilon_{5}\left\|\left(\boldsymbol{\theta}_{h}, 0\right)\right\|_{\mathbf{0}}^{2}+\frac{C_{19} h}{\epsilon_{5}}\|\mathbf{u}\|_{1, \infty, \Omega}^{2}\left\|\boldsymbol{\theta}_{h}\right\|_{0, \Omega}^{2} .
\end{aligned}
$$

For the next term, we have

$$
\begin{aligned}
T_{5,3} \leq & \frac{1}{2}\left(\left\|\boldsymbol{\theta}^{\pi} \cdot \mathbf{n}\right\|_{0, \partial \Omega}+\left\|\boldsymbol{\theta}_{h} \cdot \mathbf{n}\right\|_{0, \partial \Omega}\right)\left\|\Pi_{h}^{k}\left(\mathbf{u} \cdot \boldsymbol{\theta}_{h}\right)-\mathbf{u} \cdot \boldsymbol{\theta}_{h}\right\|_{0, \partial \Omega} \\
\leq & \frac{1}{2}\left(\left\|\left(\boldsymbol{\theta}^{\pi}, 0\right)\right\|_{\mathbf{0}}+\left\|\left(\boldsymbol{\theta}_{h}, 0\right)\right\|_{\mathbf{0}}\right)\left(\sum_{K \in \mathcal{T}_{h}}\left\|\Pi_{h}^{k}\left(\mathbf{u} \cdot \boldsymbol{\theta}_{h}\right)-\mathbf{u} \cdot \boldsymbol{\theta}_{h}\right\|_{0, \partial K \cap \partial \Omega}^{2}\right)^{\frac{1}{2}} \\
\leq & C_{20}\left(\|\left(\boldsymbol{\theta}^{\pi}, 0\right)\left[\|\|\left(\boldsymbol{\theta}_{h}, 0\right) \|_{\mathbf{0}}\right)\right. \\
& {\left[\sum_{K \in \mathcal{T}_{h}}\left\|\Pi_{h}^{k}\left(\mathbf{u} \cdot \boldsymbol{\theta}_{h}\right)-\Pi_{h}^{k} \mathbf{u} \cdot \boldsymbol{\theta}_{h}\right\|_{0, \partial K \cap \partial \Omega}^{2}+\sum_{K \in \mathcal{T}_{h}}\left\|\left(\Pi_{h}^{k} \mathbf{u}-\mathbf{u}\right) \cdot \boldsymbol{\theta}_{h}\right\|_{0, \partial K \cap \partial \Omega}^{2}\right]^{\frac{1}{2}} . }
\end{aligned}
$$

Thus, using the trace inequality (25), we have

$$
\begin{aligned}
T_{5,3} \leq & C_{21}\left(\left\|\left(\boldsymbol{\theta}^{\pi}, 0\right)\right\|+\left\|\left(\boldsymbol{\theta}_{h}, 0\right)\right\|_{\mathbf{0}}\right) \\
& {\left[\sum_{K \in \mathcal{T}_{h}} h_{K}^{-1}\left\|\Pi_{h}^{k}\left(\mathbf{u} \cdot \boldsymbol{\theta}_{h}\right)-\Pi_{h}^{k} \mathbf{u} \cdot \boldsymbol{\theta}_{h}\right\|_{0, K}^{2}+\sum_{K \in \mathcal{T}_{h}}\left\|\left(\Pi_{h}^{k} \mathbf{u}-\mathbf{u}\right)\right\|_{0, \infty, K}^{2} h_{K}^{-1}\left\|\boldsymbol{\theta}_{h}\right\|_{0, K}^{2}\right]^{\frac{1}{2}} } \\
\leq & C_{22} h^{-\frac{1}{2}}\left(\left\|\left(\boldsymbol{\theta}^{\pi}, 0\right)\right\|+\left\|\left(\boldsymbol{\theta}_{h}, 0\right)\right\|_{\mathbf{0}}\right) \\
& \left(\left\|\Pi_{h}^{k}\left(\mathbf{u} \cdot \boldsymbol{\theta}_{h}\right)-\Pi_{h}^{k} \mathbf{u} \cdot \boldsymbol{\theta}_{h}\right\|_{0, \Omega}+\left\|\left(\Pi_{h}^{k} \mathbf{u}-\mathbf{u}\right)\right\|_{0, \infty, \Omega}\left\|\boldsymbol{\theta}_{h}\right\|_{0, \Omega}\right) .
\end{aligned}
$$


Now, using Corollary 1 and (22), we conclude that

$$
\begin{aligned}
T_{5,3} & \leq C_{23}\left(\left\|\left(\boldsymbol{\theta}^{\pi}, 0\right)\right\|+\left\|\left(\boldsymbol{\theta}_{h}, 0\right)\right\|_{\mathbf{0}}\right) h^{\frac{1}{2}}\|\mathbf{u}\|_{1, \infty, \Omega}\left\|\boldsymbol{\theta}_{h}\right\|_{0, \Omega} \\
& \leq C_{24} \epsilon_{6}\left(\left\|\left(\boldsymbol{\theta}^{\pi}, 0\right)\right\|^{2}+\left\|\left(\boldsymbol{\theta}_{h}, 0\right)\right\|_{\mathbf{0}}^{2}\right)+\frac{C_{24} h}{\epsilon_{6}}\|\mathbf{u}\|_{1, \infty, \Omega}^{2}\left\|\boldsymbol{\theta}_{h}\right\|_{0, \Omega}^{2} .
\end{aligned}
$$

For $T_{5,4}$ we first have,

$$
\begin{aligned}
T_{5,4}= & \sum_{K \in \mathcal{T}_{h}{ }_{\partial}} h_{K}^{2} \llbracket \nabla p_{h} \rrbracket: \llbracket \nabla \Pi_{h}^{k}\left(\mathbf{u} \cdot \boldsymbol{\theta}_{h}\right) \rrbracket \mathrm{d} \boldsymbol{s} \\
\leq & C_{25} j\left(p_{h}, p_{h}\right)^{\frac{1}{2}}\left[\sum_{K \in \mathcal{T}_{h} K} \int_{K} h_{K}^{2} \llbracket \nabla\left(\Pi_{h}^{k}\left(\mathbf{u} \cdot \boldsymbol{\theta}_{h}\right)-\Pi_{h}^{k} \mathbf{u} \cdot \boldsymbol{\theta}_{h}\right) \rrbracket^{2} \mathrm{~d} \boldsymbol{s}\right. \\
& \left.+\sum_{K \in \mathcal{T}_{h} \int_{K}} h_{K}^{2} \llbracket \nabla\left(\Pi_{h}^{k} \mathbf{u} \cdot \boldsymbol{\theta}_{h}\right) \rrbracket^{2} \mathrm{~d} \boldsymbol{s}\right]^{\frac{1}{2}} \\
\leq & C_{26} j\left(p_{h}, p_{h}\right)^{\frac{1}{2}}\left[\sum_{K \in \mathcal{T}_{h}} h_{K}^{2}\left\|\nabla\left(\Pi_{h}^{k}\left(\mathbf{u} \cdot \boldsymbol{\theta}_{h}\right)-\Pi_{h}^{k} \mathbf{u} \cdot \boldsymbol{\theta}_{h}\right)\right\|_{0, \partial K}^{2}\right. \\
& \left.+\sum_{K \in \mathcal{T}_{h} \int_{\partial K}} h_{K}^{2}\left(\left|\boldsymbol{\theta}_{h}\right|^{2} \llbracket \nabla\left(\Pi_{h}^{k} \mathbf{u}\right) \rrbracket^{2}+\left|\Pi_{h}^{k} \mathbf{u}\right|^{2} \llbracket \nabla \boldsymbol{\theta}_{h} \rrbracket^{2}\right) \mathrm{d} \boldsymbol{s}\right]^{\frac{1}{2}} .
\end{aligned}
$$

Thus, by combining the trace inequality (25) with an inverse estimate (16), Corollary 1 and the stability estimate for the $L^{2}$-projection (20)-(21), we get

$$
\begin{aligned}
T_{5,4} \leq & C_{27} j\left(p_{h}, p_{h}\right)^{\frac{1}{2}}\left[\sum_{K \in \mathcal{T}_{h}} h_{K}^{-1}\left\|\Pi_{h}^{k}\left(\mathbf{u} \cdot \boldsymbol{\theta}_{h}\right)-\Pi_{h}^{k} \mathbf{u} \cdot \boldsymbol{\theta}_{h}\right\|_{0, K}^{2}\right. \\
& \left.+\sum_{K \in \mathcal{T}_{h}}\left\|\nabla \Pi_{h}^{k} \mathbf{u}\right\|_{0, \infty, \Omega}^{2} h_{K}\left\|\boldsymbol{\theta}_{h}\right\|_{0, K}^{2}+\left\|\Pi_{h}^{k} \mathbf{u}\right\|_{0, \infty, \Omega}^{2} j\left(\boldsymbol{\theta}_{h}, \boldsymbol{\theta}_{h}\right)\right]^{\frac{1}{2}} \\
\leq & C_{28} \epsilon_{7}\left(j\left(\Pi_{h}^{k} p, \Pi_{h}^{k} p\right)+j\left(y_{h}, y_{h}\right)\right)+\frac{C_{28}}{\epsilon_{7}} h\|\mathbf{u}\|_{1, \infty, \Omega}^{2}\left\|\boldsymbol{\theta}_{h}\right\|_{0, \Omega}^{2} \\
& +\frac{C_{\pi}^{2}}{2 \epsilon_{7}}\|\mathbf{u}\|_{0, \infty, \Omega}^{2} j\left(\boldsymbol{\theta}_{h}, \boldsymbol{\theta}_{h}\right) \\
\leq & C_{28} \epsilon_{7}\left(j\left(\Pi_{h}^{k} p, \Pi_{h}^{k} p\right)+\left\|\left(\mathbf{0}, y_{h}\right)\right\|_{\mathbf{0}}\right)+\frac{C_{28}}{\epsilon_{7}} h\|\mathbf{u}\|_{1, \infty, \Omega}^{2}\left\|\boldsymbol{\theta}_{h}\right\|_{0, \Omega}^{2} \\
& +\frac{C_{\pi}^{2}}{2 \epsilon_{7}}\|\mathbf{u}\|_{0, \infty, \Omega}^{2} j\left(\boldsymbol{\theta}_{h}, \boldsymbol{\theta}_{h}\right) .
\end{aligned}
$$


Finally, for the last term in (44), using (43), we have

$$
\begin{aligned}
& T_{6}=\sum_{K \in \mathcal{T}_{h}{ }_{\partial K}} \int_{K}^{2}\left|\mathcal{I}_{h}^{1} \mathbf{u}_{h} \cdot \mathbf{n}\right|^{2} \llbracket \nabla \Pi_{h}^{k} \mathbf{u} \rrbracket: \llbracket \nabla \boldsymbol{\theta}_{h} \rrbracket \mathrm{d} \boldsymbol{s} \\
& \leq \frac{1}{2 \epsilon_{8}} \sum_{K \in \mathcal{T}_{h \partial K}} \int_{h_{K}} h_{K}^{2}\left|\mathcal{I}_{h}^{1} \mathbf{u}_{h}\right|^{2} \llbracket \nabla \Pi_{h}^{k} \mathbf{u} \rrbracket^{2} \mathrm{~d} \boldsymbol{s}+\frac{\epsilon_{8}}{2} \sum_{K \in \mathcal{T}_{h \partial K}} \int_{h_{K}} h_{K}^{2}\left|\mathcal{I}_{h}^{1} \mathbf{u}_{h} \cdot \mathbf{n}\right|^{2} \llbracket \nabla \boldsymbol{\theta}_{h} \rrbracket^{2} \mathrm{~d} \boldsymbol{s} \\
& \leq \frac{C_{29}}{2 \epsilon_{8}}[\underbrace{\sum_{K \in \mathcal{T}_{h \partial K}} \int_{T_{K}} h_{K}^{2}\left|\mathcal{I}_{h}^{1} \mathbf{u}_{h}-\mathbf{u}_{h}\right|^{2} \llbracket \nabla \Pi_{h}^{k} \mathbf{u} \rrbracket^{2} \mathrm{~d} \boldsymbol{s}}_{T_{6,1}} \\
& +\underbrace{\left.\sum_{K \in \mathcal{T}_{h \partial K}} \int_{T_{K}} h_{K}^{2}\left(\left|\boldsymbol{\theta}_{h}\right|^{2}+\left|\Pi_{h}^{k} \mathbf{u}\right|^{2}\right) \llbracket \nabla \Pi_{h}^{k} \mathbf{u} \rrbracket^{2} \mathrm{~d} \boldsymbol{s}\right]} \\
& T_{6,2} \\
& +\frac{\epsilon_{8}}{2} \sum_{K \in \mathcal{T}_{h \partial K}} \int_{h_{K}} h_{K}^{2}\left|\mathcal{I}_{h}^{1} \mathbf{u}_{h} \cdot \mathbf{n}\right|^{2} \llbracket \nabla \boldsymbol{\theta}_{h} \rrbracket^{2} \mathrm{~d} \boldsymbol{s} .
\end{aligned}
$$

In order to estimate $T_{6,1}$, we use approximation and an inverse inequality to obtain

$$
\begin{aligned}
\left\|\mathcal{I}_{h}^{1} \mathbf{u}_{h}-\mathbf{u}_{h}\right\|_{0, \partial K}^{2} & \leq C_{30} h_{K}^{2}|\mathbf{u}|_{2, \partial K}^{2} \\
& =C_{30} h_{K}^{2}\left|\mathcal{I}_{h}^{1} \Pi_{h}^{k} \mathbf{u}-\mathbf{u}_{h}\right|_{2, \partial K}^{2} \\
& \leq C_{31}\left\|\mathcal{I}_{h}^{1} \Pi_{h}^{k} \mathbf{u}-\mathbf{u}_{h}\right\|_{0, \partial K}^{2} .
\end{aligned}
$$

This estimate, combined with (21), the trace inequality (25), approximation and an inverse inequality (16), leads to

$$
\begin{aligned}
& \int_{\partial K} h_{K}^{2}\left|\mathcal{I}_{h}^{1} \mathbf{u}_{h}-\mathbf{u}_{h}\right|^{2} \llbracket \nabla \Pi_{h}^{k} \mathbf{u} \rrbracket^{2} \mathrm{~d} s \leq\left\|\llbracket \nabla \Pi_{h}^{k} \mathbf{u} \rrbracket\right\|_{0, \infty, K}^{2} \int_{\partial K} h_{K}^{2}\left|\mathcal{I}_{h}^{1} \mathbf{u}_{h}-\mathbf{u}_{h}\right|^{2} \mathrm{~d} \boldsymbol{s} \\
& \quad \leq C_{32}\left\|\llbracket \nabla \Pi_{h}^{k} \mathbf{u} \rrbracket\right\|_{0, \infty, \partial K}^{2} \int_{\partial K} h_{K}^{2}\left|\mathcal{I}_{h}^{1} \Pi_{h}^{k} \mathbf{u}-\mathbf{u}_{h}\right|^{2} \mathrm{~d} \boldsymbol{s} \\
& \quad \leq C_{33}\left\|\llbracket \nabla \Pi_{h}^{k} \mathbf{u} \rrbracket\right\|_{0, \infty, \partial K}^{2} \int_{\partial K} h_{K}^{2}\left(\left|\mathcal{I}_{h}^{1} \Pi_{h}^{k} \mathbf{u}-\Pi_{h}^{k} \mathbf{u}\right|^{2}+\left|\boldsymbol{\theta}_{h}\right|^{2}\right) \mathrm{d} \boldsymbol{s} \\
& \leq C_{34} h_{K}\left\|\llbracket \nabla \Pi_{h}^{k} \mathbf{u} \rrbracket\right\|_{0, \infty, \partial K}^{2}\left(\left\|\mathcal{I}_{h}^{1} \Pi_{h}^{k} \mathbf{u}-\Pi_{h}^{k} \mathbf{u}\right\|_{0, K}^{2}+\left\|\boldsymbol{\theta}_{h}\right\|_{0, K}^{2}\right) \\
& \leq C_{35} h_{K}\left\|\llbracket \nabla \Pi_{h}^{k} \mathbf{u} \rrbracket\right\|_{0, \infty, \partial K}^{2}\left(h_{K}^{d}\left\|\mathcal{I}_{h}^{1} \Pi_{h}^{k} \mathbf{u}-\Pi_{h}^{k} \mathbf{u}\right\|_{0, \infty, K}^{2}+\left\|\boldsymbol{\theta}_{h}\right\|_{0, K}^{2}\right) \\
& \leq C_{36} h_{K}\left\|\llbracket \nabla \Pi_{h}^{k} \mathbf{u} \rrbracket\right\|_{0, \infty, \partial K}^{2}\left(h^{d+2}\left\|\Pi_{h}^{k} \mathbf{u}\right\|_{1, \infty, \Omega}^{2}+\left\|\boldsymbol{\theta}_{h}\right\|_{0, K}^{2}\right) \\
& \leq C_{37} h_{K}^{2-d}\left\|\llbracket \nabla \Pi_{h}^{k} \mathbf{u} \rrbracket\right\|_{0, \partial K}^{2} h^{d+2}\|\mathbf{u}\|_{1, \infty, \Omega}^{2}+h_{K} C_{37}\|\nabla \mathbf{u}\|_{1, \infty, \Omega}^{2}\left\|\boldsymbol{\theta}_{h}\right\|_{0, K}^{2} .
\end{aligned}
$$


Therefore,

$$
T_{6,1} \leq C_{38}\|\mathbf{u}\|_{1, \infty, \Omega}^{2}\left(h^{2} j\left(\Pi_{h}^{k} \mathbf{u}, \Pi_{h}^{k} \mathbf{u}\right)+h\left\|\boldsymbol{\theta}_{h}\right\|_{0, \Omega}^{2}\right)
$$

Using the trace inequality (25) and the stability of the $L^{2}$-projection (21), we obtain

$$
T_{6,2} \leq h\|\mathbf{u}\|_{1, \infty, \Omega}^{2}\left\|\boldsymbol{\theta}_{h}\right\|_{0, \Omega}^{2}+\|\mathbf{u}\|_{0, \infty, \Omega}^{2} j\left(\Pi_{h}^{k} \mathbf{u}, \Pi_{h}^{k} \mathbf{u}\right)
$$

Finally, by inserting these last two estimates into (45), we obtain

$$
\begin{aligned}
T_{6} \leq & \frac{C_{39}}{\epsilon_{8}}\left[h\|\mathbf{u}\|_{1, \infty, \Omega}^{2}\left\|\boldsymbol{\theta}_{h}\right\|_{0, \Omega}^{2}+\left(\|\mathbf{u}\|_{0, \infty, \Omega}^{2}+h^{2}\|\mathbf{u}\|_{1, \infty, \Omega}^{2}\right) j\left(\Pi_{h}^{k} \mathbf{u}, \Pi_{h}^{k} \mathbf{u}\right)\right] \\
& +\frac{\epsilon_{8}}{2}\left\|\left(\boldsymbol{\theta}_{h}, 0\right)\right\|_{\mathbf{u}_{h}}^{2} .
\end{aligned}
$$

Based on (44) and the previous estimates, we chose $\epsilon_{i}, i=1, \ldots, 8$, such that

$$
\begin{aligned}
& \frac{\epsilon_{1} C_{1}}{2}=\frac{\epsilon_{2} \gamma_{1}}{2}=\epsilon_{3} C_{15}\|\nabla \mathbf{u}\|_{0, \infty, \Omega}=\epsilon_{4} C_{17}=\epsilon_{5} C_{19}=\epsilon_{6} C_{24}=\frac{C_{\mathrm{A}}}{16}, \\
& \epsilon_{7} C_{28}=\frac{\epsilon_{8}}{2}=\frac{C_{\mathrm{A}}}{16}
\end{aligned}
$$

and $\gamma$ such as

$$
\gamma>\frac{C_{\pi}^{2}}{2 \epsilon_{7}}\|\mathbf{u}\|_{0, \infty, \Omega}^{2},
$$

for instance

$$
\gamma \stackrel{\text { def }}{=} \frac{8 C_{\pi}^{2}}{C_{\mathrm{A}}} C_{28}\|\mathbf{u}\|_{0, \infty, \Omega}^{2}+1 .
$$

Then, from (44) using the previous estimates, we get

$$
\begin{aligned}
\frac{1}{2} & \frac{\mathrm{d}}{\mathrm{d} t}\left\|\boldsymbol{\theta}_{h}\right\|_{0,2}^{2}+\frac{C_{\mathrm{A}}}{2}\left\|\left(\boldsymbol{\theta}_{h}, y_{h}\right)\right\|_{\mathbf{u}_{h}}^{2} \\
\leq & C_{40}\left(h\|\mathbf{u}\|_{1, \infty, \Omega}^{2}+\|\mathbf{u}\|_{1, \infty, \Omega}\right)\left\|\boldsymbol{\theta}_{h}\right\|_{0, \Omega}^{2} \\
& +C_{40}\left\|\left(\boldsymbol{\theta}^{\pi}, 0\right)\right\|^{2}\left(1+h^{\frac{1}{2}}\|\nabla \mathbf{u}\|_{0, \infty, \Omega}\right) \\
& +C_{40}\left(1+\|\mathbf{u}\|_{0, \infty, \Omega}^{2}+h^{2}\|\mathbf{u}\|_{1, \infty, \Omega}^{2}\right) \mathbf{J}\left[\mathbf{0} ;\left(\Pi_{h}^{k} \mathbf{u}, \Pi_{h}^{k} p\right),\left(\Pi_{h}^{k} \mathbf{u}, \Pi_{h}^{k} p\right)\right],
\end{aligned}
$$


a.e. in $(0, T)$. Therefore, using Gronwall's lemma we obtain

$$
\begin{aligned}
& \left\|\boldsymbol{\theta}_{h}\right\|_{L^{\infty}\left(0, T ; L^{2}(\Omega)\right)}^{2}+\int_{0}^{T}\left\|\left(\boldsymbol{\theta}_{h}, y_{h}\right)\right\|_{\mathbf{u}_{h}}^{2} \mathrm{~d} t \leq C_{\exp }\left\|\boldsymbol{\theta}_{h}(0)\right\|_{0, \Omega}^{2} \\
& +C_{\exp }\left[c_{1} \int_{0}^{T}\left\|\left(\boldsymbol{\theta}^{\pi}, 0\right)\right\|^{2} \mathrm{~d} t+c_{2} \int_{0}^{T} \mathbf{J}\left[\mathbf{0} ;\left(\Pi_{h}^{k} \mathbf{u}, \Pi_{h}^{k} p\right),\left(\Pi_{h}^{k} \mathbf{u}, \Pi_{h}^{k} p\right)\right] \mathrm{d} t\right],
\end{aligned}
$$

with

$$
\begin{aligned}
C_{\text {exp }} & \stackrel{\text { def }}{=} e^{C_{40} T\left(h\left\|\mathbf{u}_{L^{\infty}\left(0, T ; W^{1, \infty}(\Omega)\right)}^{2}+\right\| \mathbf{u}_{L^{\infty}\left(0, T ; W^{1, \infty}(\Omega)\right)}\right)}, \\
c_{1} & \stackrel{\text { def }}{=} C_{40}\left(1+h^{\frac{1}{2}}\|\mathbf{u}\|_{L^{\infty}\left(0, T ; W^{1, \infty}(\Omega)\right)}\right), \\
c_{2} & \stackrel{\text { def }}{=} C_{40}\left(1+\|\mathbf{u}\|_{L^{\infty}\left(0, T ; L^{\infty}(\Omega)\right)}^{2}+h^{2}\|\mathbf{u}\|_{L^{\infty}\left(0, T ; W^{1, \infty}(\Omega)\right)}^{2}\right),
\end{aligned}
$$

which gives (40) and (41).

\subsection{Pressure estimates}

Based on the previous convergence analysis for the velocity and on the modified infsup condition (35), in this paragraph, we provide error estimates for the pressure. The optimal approach to follow here is not clear cut. Depending on how much regularity one can expect for the pressure, different analysis should be applied. Here we choose first to present two error estimates for the pressure in the $L^{2}$-norm and, for regular pressures, in the $H^{1}$-norm. The upper bounds consist of one part using the previous convergence of the velocities, and a second part consisting of different norms of the approximation error in the time derivative of the velocities. We will then show how to get an estimate that is optimal for low-order elements if the pressure is in $H^{1}\left(0, T ; H^{1}(\Omega)\right)$ using a suboptimal approximation of the time derivative of the velocities. Other possible strategies will be briefly discussed in the conclusion.

Lemma 10 Let $(\mathbf{u}, p)$ the solution of (1), $\left(\mathbf{u}_{h}, p_{h}\right) \in W_{h}^{k}$ the solution of (9), with $\mathbf{u}_{0, h}=P_{h}^{k} \mathbf{u}_{0}$, and assume that $(\mathbf{u}, p)$ has the regularity (5) and that $v<h$. Then, the following error estimate holds

$$
\begin{aligned}
\int_{0}^{T}\left\|p-p_{h}\right\|_{0, \Omega}^{2} \mathrm{~d} t \leq C & {\left[h^{2\left(r_{\mathbf{u}}-1\right)}\|\mathbf{u}\|_{L^{2}\left(0, T ; H^{r} \mathbf{u}(\Omega)\right)}^{2}+h^{2\left(r_{p}-1\right)}\|p\|_{L^{2}\left(0, T ; H^{r}(\Omega)\right)}^{2}\right.} \\
& \left.+\left\|\partial_{t}\left(\mathbf{u}-\mathbf{u}_{h}\right)\right\|_{L^{2}\left(0, T ; V^{\prime}(\Omega)\right)}^{2}\right] .
\end{aligned}
$$

with $V^{\prime}(\Omega)$ standing for the dual of $H^{1}(\Omega)$ and $C>0$ a constant independent of $v$ and $h$. 
Proof From (42) and (19), we only need to estimate

$$
\int_{0}^{T}\left\|y_{h}\right\|_{0, \Omega}^{2} \mathrm{~d} t .
$$

To this aim, we use the modified inf-sup condition (35). Let $\mathbf{v}_{h} \in\left[V_{h}^{k}\right]^{d}$, from (43), we have

$$
b_{h}\left(y_{h}, \mathbf{v}_{h}\right)=b_{h}\left(p-p_{h}, \mathbf{v}_{h}\right)-b_{h}\left(y^{\pi}, \mathbf{v}_{h}\right) .
$$

The last term can be bound using Lemma 9. This yields

$$
\left|b_{h}\left(y^{\pi}, \mathbf{v}_{h}\right)\right| \leq C \mid\left\|\left(\mathbf{0}, y^{\pi}\right) \llbracket\right\|\left\|\mathbf{v}_{h}\right\|_{1, \Omega} .
$$

On the other hand, using the approximate Galerkin orthogonality (Lemma 1) with $q_{h}=0$, for the first term we get

$$
\begin{aligned}
b_{h}\left(p_{h}-p, \mathbf{v}_{h}\right)= & \underbrace{a_{h}\left(\mathbf{u}-\mathbf{u}_{h}, \mathbf{v}_{h}\right)-\gamma j\left(\mathbf{u}_{h}, \mathbf{v}_{h}\right)}_{T_{1}} \\
& +\underbrace{c_{h}\left(\mathbf{u} ; \mathbf{u}, \mathbf{v}_{h}\right)-c_{h}\left(\mathbf{u}_{h} ; \mathbf{u}_{h}, \mathbf{v}_{h}\right)}_{T_{2}} \\
& -\underbrace{j_{\mathbf{u}_{h}\left(\mathbf{u}_{h}, \mathbf{v}_{h}\right)}}_{T_{3}}+\underbrace{\left(\partial_{t}\left(\mathbf{u}-\mathbf{u}_{h}\right), \mathbf{v}_{h}\right)}_{T_{4}} .
\end{aligned}
$$

Term $T_{1}$ is treated using the arguments given in [10, Proof of Theorem 4.4]. Thus, we have

$$
\begin{aligned}
T_{1} \leq & C\left\|\left(\mathbf{u}-\mathbf{u}_{h}, 0\right)\right\|_{\mathbf{0}}\left\|\left(\mathbf{v}_{h}, 0\right)\right\|_{\mathbf{0}} \\
& \underbrace{-\left\langle 2 \nu \boldsymbol{\varepsilon}\left(\mathbf{u}-\mathbf{u}_{h}\right) \mathbf{n}, \mathbf{v}_{h}\right\rangle_{\partial \Omega}-\left\langle\mathbf{u}-\mathbf{u}_{h}, 2 \nu \boldsymbol{\varepsilon}\left(\mathbf{v}_{h}\right) \mathbf{n}\right\rangle_{\partial \Omega}}_{T_{1,1}} .
\end{aligned}
$$

The boundary terms are controlled in the following fashion

$$
\begin{aligned}
T_{1,1} \leq & 2\left\|(v h)^{\frac{1}{2}} \boldsymbol{\varepsilon}\left(\mathbf{u}-\mathbf{u}_{h}\right)\right\|_{0, \partial \Omega}\left\|v^{\frac{1}{2}} h^{-\frac{1}{2}} \mathbf{v}_{h}\right\|_{0, \partial \Omega} \\
& +2\left\|(v h)^{\frac{1}{2}} \boldsymbol{\varepsilon}\left(\mathbf{v}_{h}\right)\right\|_{0, \partial \Omega}\left\|v^{\frac{1}{2}} h^{-\frac{1}{2}}\left(\mathbf{u}-\mathbf{u}_{h}\right)\right\|_{0, \partial \Omega} \\
\leq & 2\left\|(v h)^{\frac{1}{2}} \boldsymbol{\varepsilon}\left(\mathbf{u}-\mathbf{u}_{h}\right)\right\|_{0, \partial \Omega}\left\|\left(\mathbf{v}_{h}, 0\right)\right\|_{\mathbf{0}} \\
& +2\left\|(v h)^{\frac{1}{2}} \boldsymbol{\varepsilon}\left(\mathbf{v}_{h}\right)\right\|_{0, \partial \Omega}\left\|\left(\mathbf{u}-\mathbf{u}_{h}, 0\right)\right\|_{\mathbf{0}} .
\end{aligned}
$$


On the other hand, we have

$$
\begin{aligned}
\left\|(\nu h)^{\frac{1}{2}} \boldsymbol{\varepsilon}\left(\mathbf{u}-\mathbf{u}_{h}\right)\right\|_{0, \partial \Omega} \leq & \left\|(v h)^{\frac{1}{2}} \boldsymbol{\varepsilon}\left(\mathbf{u}-\Pi_{h}^{k} \mathbf{u}\right)\right\|_{0, \partial \Omega} \\
& +\left\|(v h)^{\frac{1}{2}} \boldsymbol{\varepsilon}\left(\Pi_{h}^{k} \mathbf{u}-\mathbf{u}_{h}\right)\right\|_{0, \partial \Omega},
\end{aligned}
$$

where the first term satisfies, using the trace inequality (24), (19) and that $v<h$,

$$
\begin{aligned}
\left\|(\nu h)^{\frac{1}{2}} \boldsymbol{\varepsilon}\left(\mathbf{u}-\Pi_{h}^{k} \mathbf{u}\right)\right\|_{0, \partial \Omega} & \leq C v^{\frac{1}{2}} h^{r_{\mathbf{u}}-1}\|\mathbf{u}\|_{r_{\mathbf{u}}, \Omega} \\
& \leq C h^{r_{\mathbf{u}}-\frac{1}{2}}\|\mathbf{u}\|_{r_{\mathbf{u}}, \Omega},
\end{aligned}
$$

and the second, using (25),

$$
\begin{aligned}
\left\|(\nu h)^{\frac{1}{2}} \boldsymbol{\varepsilon}\left(\Pi_{h}^{k} \mathbf{u}-\mathbf{u}_{h}\right)\right\|_{0, \partial \Omega} & \leq C\left\|v^{\frac{1}{2}} \boldsymbol{\varepsilon}\left(\Pi_{h}^{k} \mathbf{u}-\mathbf{u}_{h}\right)\right\|_{0, \Omega} \\
& \leq C\left\|\left(\Pi_{h}^{k} \mathbf{u}-\mathbf{u}_{h}, 0\right)\right\|_{\mathbf{0}} .
\end{aligned}
$$

In the same fashion we conclude that

$$
\left\|(\nu h)^{\frac{1}{2}} \boldsymbol{\varepsilon}\left(\mathbf{v}_{h}\right)\right\|_{0, \partial \Omega} \leq C\left\|\left(\mathbf{v}_{h}, 0\right)\right\|_{\mathbf{0}} .
$$

Thus, collecting terms, using the fact that $\left\|\left(\mathbf{v}_{h}, 0\right)\right\|_{0} \leq C\left\|\mathbf{v}_{h}\right\|_{1, \Omega}$ since $v<h$, we have

$$
T_{1} \leq C\left(\left\|\left(\mathbf{u}-\mathbf{u}_{h}, 0\right)\right\|_{\mathbf{0}}+h^{r_{\mathbf{u}}-\frac{1}{2}}\|\mathbf{u}\|_{r_{\mathbf{u}}, \Omega}\right)\left\|\mathbf{v}_{h}\right\|_{1, \Omega}
$$

For the third term, we have

$$
\begin{aligned}
T_{2}= & \left(\mathbf{u} \cdot \nabla\left(\mathbf{u}-\mathbf{u}_{h}\right), \mathbf{v}_{h}\right)+\left(\left(\mathbf{u}-\mathbf{u}_{h}\right) \cdot \nabla \mathbf{u}_{h}, \mathbf{v}_{h}\right) \\
& +\frac{1}{2}\left\langle\mathbf{u}_{h} \cdot \mathbf{n} \mathbf{u}_{h}, \mathbf{v}_{h}\right\rangle_{\partial \Omega}-\frac{1}{2}\left(\nabla \cdot \mathbf{u}_{h}, \mathbf{u}_{h} \cdot \mathbf{v}_{h}\right) .
\end{aligned}
$$

Thus, integrating by parts and since $\nabla \cdot \mathbf{u}=0$ and $\mathbf{u}_{\mid \partial \Omega}=\mathbf{0}$, we get

$$
\begin{array}{r}
T_{2}=\underbrace{-\left(\left(\mathbf{u}-\mathbf{u}_{h}\right) \cdot \nabla \mathbf{v}_{h}, \mathbf{u}\right)-\left(\mathbf{u}_{h} \cdot \nabla \mathbf{v}_{h}, \mathbf{u}-\mathbf{u}_{h}\right)}_{T_{2,1}} \\
-\frac{1}{2} \underbrace{\left\langle\mathbf{u}_{h} \cdot \mathbf{n} \mathbf{u}_{h}, \mathbf{v}_{h}\right\rangle_{\partial \Omega}}_{T_{2,2}}+\frac{1}{2} \underbrace{\left(\nabla \cdot \mathbf{u}_{h}, \mathbf{u}_{h} \cdot \mathbf{v}_{h}\right)}_{T_{2,3}} .
\end{array}
$$

Now, we treat each term separately. Using Cauchy-Schwarz on the first term one gets

$$
T_{2,1} \leq C\left(\|\mathbf{u}\|_{0, \infty, \Omega}+\left\|\mathbf{u}_{h}\right\|_{0, \infty, \Omega}\right)\left\|\mathbf{u}-\mathbf{u}_{h}\right\|_{0, \Omega}\left\|\mathbf{v}_{h}\right\|_{1, \Omega}
$$


For the second, using the Cauchy-Schwarz inequality and the argument followed in (33), we obtain

$$
\begin{aligned}
T_{2,2} & \leq\left\|\mathbf{u}_{h}\right\|_{0, \infty, \Omega}\left\|\mathbf{u}_{h} \cdot \mathbf{n}\right\|_{0,2 \Omega}\left\|\mathbf{v}_{h}\right\|_{0,2 \Omega} \\
& \leq C\left\|\mathbf{u}_{h}\right\|_{0, \infty, \Omega}\left\|\left(\mathbf{u}-\mathbf{u}_{h}, p-p_{h}\right)\right\|_{\mathbf{u}_{h}}\left\|\mathbf{v}_{h}\right\|_{1, \Omega} .
\end{aligned}
$$

Using the Cauchy-Schwarz inequality, in the third term, we have

$$
\begin{aligned}
T_{2,3} & \leq h^{-\frac{1}{2}}\left\|h^{\frac{1}{2}} \nabla \cdot \mathbf{u}_{h}\right\|_{0, \Omega}\left\|\mathbf{u}_{h}\right\|_{0, \infty, \Omega}\left\|\mathbf{v}_{h}\right\|_{0, \Omega} \\
& \leq C h^{-\frac{1}{2}}\left\|\mathbf{u}_{h}\right\|_{0, \infty, \Omega}\left\|\left(\mathbf{u}-\mathbf{u}_{h}, p-p_{h}\right)\right\|_{\mathbf{u}_{h}}\left\|\mathbf{v}_{h}\right\|_{1, \Omega} .
\end{aligned}
$$

For the jump term in (47), we use the Cauchy-Schwarz inequality, (16) and the regularity $\mathbf{u} \in H^{r_{\mathbf{u}}}(\Omega)$, which yields

$$
\begin{aligned}
T_{3} & \leq j_{\mathbf{u}_{h}}\left(\mathbf{u}_{h}, \mathbf{u}_{h}\right)^{\frac{1}{2}} j_{\mathbf{u}_{h}}\left(\mathbf{v}_{h}, \mathbf{v}_{h}\right)^{\frac{1}{2}} \\
& \leq j_{\mathbf{u}_{h}}\left(\mathbf{u}_{h}, \mathbf{u}_{h}\right)^{\frac{1}{2}}\left(\sum_{K \in \mathcal{T}_{h \partial K}} \int_{K} h_{K}^{2}\left|\mathbf{u}_{h} \cdot \mathbf{n}\right|^{2} \llbracket \nabla \mathbf{v}_{h} \rrbracket^{2}\right)^{\frac{1}{2}} \\
& \leq C j_{\mathbf{u}_{h}}\left(\mathbf{u}_{h}, \mathbf{u}_{h}\right)^{\frac{1}{2}}\left\|\mathbf{u}_{h}\right\|_{0, \infty, \Omega}\left(\sum_{K \in \mathcal{T}_{h}} h_{K}\left\|\nabla \mathbf{v}_{h}\right\|_{0, K}^{2}\right)^{\frac{1}{2}} \\
& \leq C h^{\frac{1}{2}} j_{\mathbf{u}_{h}}\left(\mathbf{u}_{h}, \mathbf{u}_{h}\right)^{\frac{1}{2}}\left\|\mathbf{u}_{h}\right\|_{0, \infty, \Omega}\left\|\mathbf{v}_{h}\right\|_{1, \Omega}, \\
& \leq C h^{\frac{1}{2}}\left\|\mathbf{u}_{h}\right\|_{0, \infty, \Omega}\left\|\left(\mathbf{u}-\mathbf{u}_{h}, p-p_{h}\right)\right\|_{\mathbf{u}_{h}}\left\|\mathbf{v}_{h}\right\|_{1, \Omega} .
\end{aligned}
$$

Finally by duality, we have

$$
T_{4} \leq\left\|\partial_{t}\left(\mathbf{u}-\mathbf{u}_{h}\right)\right\|_{V^{\prime}(\Omega)}\left\|\mathbf{v}_{h}\right\|_{1, \Omega}
$$

Therefore, from (47) and by collecting the previous estimations, we have

$$
\begin{aligned}
& \left|b_{h}\left(p_{h}-p, \mathbf{v}_{h}\right)\right|^{2} \leq C\left\{\left\|\partial_{t}\left(\mathbf{u}-\mathbf{u}_{h}\right)\right\|_{V^{\prime}(\Omega)}^{2}+h^{2 r_{\mathbf{u}}-1}\|\mathbf{u}\|_{r_{\mathbf{u}}, \Omega}^{2}\right. \\
& +\left(1+h^{-1}\left\|\mathbf{u}_{h}\right\|_{0, \infty, \Omega}^{2}\right)\left\|\left(\mathbf{u}-\mathbf{u}_{h}, p-p_{h}\right)\right\|_{\mathbf{u}_{h}}^{2} \\
& \left.\quad+\left(\|\mathbf{u}\|_{0, \infty, \Omega}^{2}+\left\|\mathbf{u}_{h}\right\|_{0, \infty, \Omega}^{2}\right)\left\|\mathbf{u}-\mathbf{u}_{h}\right\|_{0, \Omega}^{2}\right\}\left\|\mathbf{v}_{h}\right\|_{1, \Omega}^{2} .
\end{aligned}
$$

We conclude the proof after integration over $(0, T)$ and application of Lemma 6 and Corollaries 3 and 4 .

Remark 3 From the optimal convergence estimate provided by Corollary 3, one would expect a similar rate for the pressure. However, the fact that, at the discrete level, the convective velocity is not divergence free, leads to a loss of half an order in the pressure 
estimate, see Eq. (49). The reason for this is that we do not have stability of the gradient of the finite element solution in $W^{1, \infty}(\Omega)$. However, if the solution is sufficiently regular and the polynomial order is sufficiently high we can use an argument similar to that of term $T_{5}$ of Eq. (44) to improve the estimate.

Unfortunately, it seems difficult to obtain an estimate of the time derivative of the velocities in the dual norm of $H^{1}$ (appearing in the previous Lemma). As pointed out in [25], one may obtain a crude bound by using, instead, the $L^{2}$-norm of the error in the time derivative. We therefore propose to estimate the error in the gradients of the pressure, valid only if the pressure is sufficiently regular, but leading to an estimate which is close to optimal and which uses the $L^{2}$-norm of the error in the time derivative. In fact as in the analysis for the velocities only a factor $h^{\frac{1}{2}}$ is lost.

Lemma 11 Under the hypothesis of the previous corollary, the following error estimate holds

$$
\begin{aligned}
& \int_{0}^{T}\left\|\nabla\left(p-p_{h}\right)\right\|_{0, \Omega}^{2} \mathrm{~d} t \leq\left[h^{2 r_{\mathbf{u}}-3}\|\mathbf{u}\|_{L^{2}\left(0, T ; H^{r} \mathbf{u}(\Omega)\right)}^{2}\right. \\
& \left.\quad+h^{2\left(r_{p}-1\right)}\|p\|_{L^{2}\left(0, T ; H^{r p}(\Omega)\right)}^{2}+\left\|\partial_{t}\left(\mathbf{u}-\mathbf{u}_{h}\right)\right\|_{L^{2}\left(0, T ; L^{2}(\Omega)\right)}^{2}\right]
\end{aligned}
$$

Proof To estimate the error in the pressure gradient we start by noticing that

$$
\begin{aligned}
& \int_{0}^{T}\left\|\nabla\left(p-p_{h}\right)\right\|_{0, \Omega}^{2} \mathrm{~d} t \leq C \int_{0}^{T}\left\|\nabla p-\Pi_{h}^{k}(\nabla p)\right\|_{0, \Omega}^{2} \mathrm{~d} t \\
& \quad+C \int_{0}^{T}\left\|\Pi_{h}^{k}(\nabla p)-\Pi_{h}^{k}\left(\nabla p_{h}\right)\right\|_{0, \Omega}^{2} \mathrm{~d} t+C \int_{0}^{T}\left\|\Pi_{h}^{k}\left(\nabla p_{h}\right)-\nabla p_{h}\right\|_{0, \Omega}^{2} \mathrm{~d} t .
\end{aligned}
$$

Using approximation, (28), the fact that $p \in H^{s}(\Omega)$ and Corollary 3, we obtain

$$
\begin{aligned}
& \int_{0}^{T}\left\|\nabla p-\Pi_{h}^{k}(\nabla p)\right\|_{0, \Omega}^{2}+\int_{0}^{T}\left\|\Pi_{h}^{k}\left(\nabla p_{h}\right)-\nabla p_{h}\right\|_{0, \Omega}^{2} \\
& \quad \leq C h^{2\left(r_{p}-1\right)}\|p\|_{L^{2}\left(0, T ; H^{r p}(\Omega)\right)}^{2}+C h^{-1} \int_{0}^{T} j\left(p_{h}, p_{h}\right) \mathrm{d} t \\
& =C h^{2\left(r_{p}-1\right)}\|p\|_{L^{2}\left(0, T ; H^{r}(\Omega)\right)}^{2}+C h^{-1} \int_{0}^{T} j\left(p-p_{h}, p-p_{h}\right) \mathrm{d} t \\
& \quad \leq C\left[h^{2\left(r_{\mathbf{u}}-1\right)}\|\mathbf{u}\|_{L^{2}\left(0, T ; H^{\left.r_{\mathbf{u}}(\Omega)\right)}\right.}^{2}+h^{2\left(r_{p}-1\right)}\|p\|_{L^{2}\left(0, T ; H^{r}(\Omega)\right)}^{2}\right] .
\end{aligned}
$$


Hence, it is sufficient to study the second term. Note that, by the orthogonality of the $L^{2}$-projection and a partial integration, we have

$$
\begin{aligned}
\left\|\Pi_{h}^{k}(\nabla p)-\Pi_{h}^{k}\left(\nabla p_{h}\right)\right\|_{0, \Omega}^{2}= & \left(\nabla p-\nabla p_{h}, \Pi_{h}^{k}(\nabla p)-\Pi_{h}^{k}\left(\nabla p_{h}\right)\right) \\
= & -\left(p-p_{h}, \nabla \cdot\left(\Pi_{h}^{k}(\nabla p)-\Pi_{h}^{k}\left(\nabla p_{h}\right)\right)\right) \\
& +\left\langle p-p_{h},\left(\Pi_{h}^{k}(\nabla p)-\Pi_{h}^{k}\left(\nabla p_{h}\right)\right) \cdot \mathbf{n}\right\rangle_{\partial \Omega} \\
= & b_{h}\left(p-p_{h}, \mathbf{v}_{h, p}^{k}\right),
\end{aligned}
$$

with the notation $\mathbf{v}_{h, p}^{k} \stackrel{\text { def }}{=} \Pi_{h}^{k}\left(\nabla p-\nabla p_{h}\right)$.

Therefore, using the approximate Galerkin orthogonality (Lemma 1) with $\left(\mathbf{v}_{h}, q_{h}\right)=$ $\left(\mathbf{v}_{h, p}^{k}, 0\right)$, we get

$$
\begin{aligned}
\left\|\Pi_{h}^{k} \nabla p-\Pi_{h}^{k} \nabla p_{h}\right\|_{0, \Omega}^{2}= & a_{h}\left(\mathbf{u}-\mathbf{u}_{h}, \mathbf{v}_{h, p}^{k}\right)-\gamma j\left(\mathbf{u}_{h}, \mathbf{v}_{h, p}^{k}\right) \\
& +c_{h}\left(\mathbf{u} ; \mathbf{u}, \mathbf{v}_{h, p}^{k}\right)-c_{h}\left(\mathbf{u}_{h} ; \mathbf{u}_{h}, \mathbf{v}_{h, p}^{k}\right) \\
& -j_{\mathbf{u}_{h}}\left(\mathbf{u}_{h}, \mathbf{v}_{h, p}^{k}\right)+\left(\partial_{t}\left(\mathbf{u}-\mathbf{u}_{h}\right), \mathbf{v}_{h, p}^{k}\right) .
\end{aligned}
$$

Proceeding term by term, in a fashion similar to the previous lemma with $\mathbf{v}_{h, p}^{k}$ instead of $\mathbf{v}_{h}$ and using an inverse inequality, we obtain

$$
\begin{aligned}
& \left\|\Pi_{h}^{k}(\nabla p)-\Pi_{h}^{k}\left(\nabla p_{h}\right)\right\|_{0, \Omega}^{2} \leq C h^{-2}\left\{h^{2 r_{p}}\|p\|_{r_{p}, \Omega}^{2}\right. \\
& \quad+h^{2 r_{\mathbf{u}}-1}\|\mathbf{u}\|_{r_{\mathbf{u}}, \Omega}^{2}+\left(1+h\left\|\mathbf{u}_{h}\right\|_{0, \infty, \Omega}^{2}\right)\left\|\left(\mathbf{u}-\mathbf{u}_{h}, p-p_{h}\right)\right\|_{\mathbf{u}_{h}}^{2} \\
& \left.\quad+\left(\|\mathbf{u}\|_{0, \infty, \Omega}^{2}+\left\|\mathbf{u}_{h}\right\|_{0, \infty, \Omega}^{2}\right)\left\|\mathbf{u}-\mathbf{u}_{h}\right\|_{0, \Omega}^{2}\right\} \\
& \quad+C\left(h^{-1}\left\|\mathbf{u}_{h}\right\|_{0, \infty, \Omega}^{2}\left\|\left(\mathbf{u}-\mathbf{u}_{h}, p-p_{h}\right)\right\|_{\mathbf{u}_{h}}^{2}+\left\|\partial_{t}\left(\mathbf{u}-\mathbf{u}_{h}\right)\right\|_{0, \Omega}^{2}\right) .
\end{aligned}
$$

Finally, we conclude the proof after integration over $(0, T)$, application of the results of Corollaries 3 and 4 and (50).

To close the problem of convergence of the pressure approximations we need an estimate of the error in the time derivative of the error. This is the subject of the next paragraph. Here we focus on proving an estimate that requires minimum assumptions on the pressure regularity, but makes the estimate of Lemma 11 quasi-optimal for piecewise linear approximations.

\subsection{An estimate for $\partial_{t}\left(\mathbf{u}-\mathbf{u}_{h}\right)$}

The following theorem states the main result of this paragraph. 
Theorem 3 Let $(\mathbf{u}, p)$ the solution of (1), $\left(\mathbf{u}_{h}, p_{h}\right) \in W_{h}^{k}$ the solution of (9), with $\mathbf{u}_{0, h}=P_{h}^{k} \mathbf{u}_{0}$, assume that $(\mathbf{u}, p)$ has the regularity (5)-(6) and that $v<h$. Then, the following estimate holds

$$
\int_{0}^{T}\left\|\partial_{t}\left(\mathbf{u}-\mathbf{u}_{h}\right)\right\|_{0, \Omega}^{2} \mathrm{~d} t \leq C(\mathbf{u}, p, T)\left(h^{2 \alpha-3}+h^{\alpha-\frac{1}{2}}\|p\|_{H^{1}\left(0, T ; H^{1}(\Omega)\right)}\right),
$$

with $\alpha \stackrel{\text { def }}{=} \min \left\{r_{\mathbf{u}}, r_{p}\right\}, C>0$ with no explicit dependence of $v$ and $h$ and $C(\mathbf{u}, p, T)$ is proportional to the sum of the the constant of Theorem 2 and the constant of the second estimate of Lemma 5.

Proof We first decompose the error $\left(\mathbf{u}-\mathbf{u}_{h}, p-p_{h}\right)$ in two parts, using the projection operator $S_{h} \stackrel{\text { def }}{=}\left(P_{h}^{k}, R_{h}^{k}\right)$ defined by (34)

$$
\begin{aligned}
& \mathbf{u}-\mathbf{u}_{h}=\underbrace{\mathbf{u}-P_{h}^{k} \mathbf{u}}_{\boldsymbol{\theta}^{\pi}}+\underbrace{P_{h}^{k} \mathbf{u}-\mathbf{u}_{h}}_{\boldsymbol{\theta}_{h}}=\boldsymbol{\theta}^{\pi}+\boldsymbol{\theta}_{h}, \\
& p-p_{h}=\underbrace{p-R_{h}^{k} \mathbf{u}}_{y^{\pi}}+\underbrace{R_{h}^{k} \mathbf{u}-p_{h}}_{y_{h}}=y^{\pi}+y_{h} .
\end{aligned}
$$

Thus, using the triangle inequality and from Lemma 5, we only need to estimate

$$
\int_{0}^{T}\left\|\partial_{t} \boldsymbol{\theta}_{h}\right\|_{0, \Omega}^{2} \mathrm{~d} t
$$

To this aim, we first test the modified Galerkin orthogonality (Lemma 1) with $\left(\mathbf{v}_{h}, q_{h}\right)=$ $\left(\partial_{t} \boldsymbol{\theta}_{h}, 0\right)$, to obtain

$$
\begin{aligned}
& \left(\partial_{t}\left(\mathbf{u}-\mathbf{u}_{h}\right), \partial_{t} \boldsymbol{\theta}_{h}\right)+a_{h}\left(\mathbf{u}-\mathbf{u}_{h}, \partial_{t} \boldsymbol{\theta}_{h}\right)+b_{h}\left(p-p_{h}, \partial_{t} \boldsymbol{\theta}_{h}\right) \\
& \quad+c_{h}\left(\mathbf{u} ; \mathbf{u}, \partial_{t} \boldsymbol{\theta}_{h}\right)-c_{h}\left(\mathbf{u}_{h} ; \mathbf{u}_{h}, \partial_{t} \boldsymbol{\theta}_{h}\right) \\
& \quad+\gamma j\left(\mathbf{u}-\mathbf{u}_{h}, \partial_{t} \boldsymbol{\theta}_{h}\right)+j_{\mathbf{u}_{h}}\left(\mathbf{u}-\mathbf{u}_{h}, \partial_{t} \boldsymbol{\theta}_{h}\right)=0 .
\end{aligned}
$$

Thus, using (52), we get

$$
\begin{aligned}
& \left\|\partial_{t} \boldsymbol{\theta}_{h}\right\|_{0, \Omega}^{2}+a_{h}\left(\boldsymbol{\theta}_{h}, \partial_{t} \boldsymbol{\theta}_{h}\right)+b_{h}\left(y_{h}, \partial_{t} \boldsymbol{\theta}_{h}\right)+\gamma j\left(\boldsymbol{\theta}_{h}, \partial_{t} \boldsymbol{\theta}_{h}\right) \\
& =-\left(\partial_{t} \boldsymbol{\theta}^{\pi}, \partial_{t} \boldsymbol{\theta}_{h}\right)-a_{h}\left(\boldsymbol{\theta}^{\pi}, \partial_{t} \boldsymbol{\theta}_{h}\right)-b_{h}\left(y^{\pi}, \partial_{t} \boldsymbol{\theta}_{h}\right)+\gamma j\left(P_{h}^{k} \mathbf{u}, \partial_{t} \boldsymbol{\theta}_{h}\right) \\
& \quad+c_{h}\left(\mathbf{u}_{h} ; \mathbf{u}_{h}, \partial_{t} \boldsymbol{\theta}_{h}\right)-c_{h}\left(\mathbf{u} ; \mathbf{u}, \partial_{t} \boldsymbol{\theta}_{h}\right)+j_{\mathbf{u}_{h}}\left(\mathbf{u}_{h}, \partial_{t} \boldsymbol{\theta}_{h}\right) .
\end{aligned}
$$

Using the definition of $a_{h}$, from (12), one readily obtains that

$$
a_{h}\left(\boldsymbol{\theta}_{h}, \partial_{t} \boldsymbol{\theta}_{h}\right)+\gamma j\left(\boldsymbol{\theta}_{h}, \partial_{t} \boldsymbol{\theta}_{h}\right)=\frac{1}{2} \partial_{t}\left[a_{h}\left(\boldsymbol{\theta}_{h}, \boldsymbol{\theta}_{h}\right)+\gamma j\left(\boldsymbol{\theta}_{h}, \boldsymbol{\theta}_{h}\right)\right] .
$$


We now test (9) with $\mathbf{v}_{h}=\mathbf{0}$, and derive the remaining equation with respect to $t$, which yields

$$
\begin{aligned}
0 & =\partial_{t}\left(b_{h}\left(q_{h}, \mathbf{u}_{h}\right)-j\left(p_{h}, q_{h}\right)\right) \\
& =b_{h}\left(q_{h}, \partial_{t} \mathbf{u}_{h}\right)-j\left(\partial_{t} p_{h}, q_{h}\right)+b_{h}\left(\partial_{t} q_{h}, \mathbf{u}_{h}\right)-j\left(p_{h}, \partial_{t} q_{h}\right) \\
& =b_{h}\left(q_{h}, \partial_{t} \mathbf{u}_{h}\right)-j\left(\partial_{t} p_{h}, q_{h}\right) .
\end{aligned}
$$

The last equality is obtained by noticing that $\partial_{t} q_{h} \in V_{h}^{k}$ and therefore

$$
b_{h}\left(\partial_{t} q_{h}, \mathbf{u}_{h}\right)-j\left(p_{h}, \partial_{t} q_{h}\right)=0 .
$$

We then have

$$
b_{h}\left(q_{h}, \partial_{t} \mathbf{u}_{h}\right)-j\left(\partial_{t} p_{h}, q_{h}\right)=0,
$$

for all $q_{h} \in V_{h}^{k}$. On the other hand, using the same argument, from (34) we obtain

$$
b_{h}\left(q_{h}, \partial_{t} P_{h}^{k} \mathbf{u}\right)=j\left(\partial_{t} R_{h}^{k} \mathbf{u}, q_{h}\right)
$$

for all $q_{h} \in V_{h}^{k}$. Thus, by taking $q_{h}=y_{h}$ in (55) and (56), we get

$$
\begin{aligned}
b_{h}\left(y_{h}, \partial_{t} \boldsymbol{\theta}_{h}\right) & =j\left(\partial_{t} y_{h}, y_{h}\right) \\
& =\frac{1}{2} \partial_{t} j\left(y_{h}, y_{h}\right) .
\end{aligned}
$$

Therefore, by inserting (54) and (57) into (53), we have

$$
\begin{aligned}
\left\|\partial_{t} \boldsymbol{\theta}_{h}\right\|_{0, \Omega}^{2}+\frac{1}{2} \partial_{t}\left[a_{h}\left(\boldsymbol{\theta}_{h}, \boldsymbol{\theta}_{h}\right)+j\left(y_{h}, y_{h}\right)+\gamma j\left(\boldsymbol{\theta}_{h}, \boldsymbol{\theta}_{h}\right)\right] \\
=\underbrace{-\left(\partial_{t} \boldsymbol{\theta}^{\pi}, \partial_{t} \boldsymbol{\theta}_{h}\right)-a_{h}\left(\boldsymbol{\theta}^{\pi}, \partial_{t} \boldsymbol{\theta}_{h}\right)-b_{h}\left(y^{\pi}, \partial_{t} \boldsymbol{\theta}_{h}\right)+\gamma j\left(P_{h}^{k} \mathbf{u}, \partial_{t} \boldsymbol{\theta}_{h}\right)}_{T_{1}} \\
+\underbrace{c_{h}\left(\mathbf{u}_{h} ; \mathbf{u}_{h}, \partial_{t} \boldsymbol{\theta}_{h}\right)-c_{h}\left(\mathbf{u} ; \mathbf{u}, \partial_{t} \boldsymbol{\theta}_{h}\right)}_{T_{2}}+\underbrace{j_{\mathbf{u}_{h}\left(\mathbf{u}_{h}, \partial_{t} \boldsymbol{\theta}_{h}\right)}}_{T_{3}} .
\end{aligned}
$$

Now we estimate the terms $T_{i}$ for $i=1, \ldots, 3$. In the following, $\epsilon>0$ stands for a constant to be fixed later on. For the first term, we use (34) to obtain,

$$
\begin{aligned}
T_{1} & =-\left(\partial_{t} \boldsymbol{\theta}^{\pi}, \partial_{t} \boldsymbol{\theta}_{h}\right)+\left(\boldsymbol{\theta}^{\pi}, \partial_{t} \boldsymbol{\theta}_{h}\right)-b_{h}\left(p, \partial_{t} \boldsymbol{\theta}_{h}\right) \\
& \leq \frac{1}{2 \epsilon}\left(\left\|\partial_{t} \boldsymbol{\theta}^{\pi}\right\|_{0, \Omega}^{2}+\left\|\boldsymbol{\theta}^{\pi}\right\|_{0, \Omega}^{2}\right)+\frac{\epsilon}{2}\left\|\partial_{t} \boldsymbol{\theta}_{h}\right\|^{2}-b_{h}\left(p, \partial_{t} \boldsymbol{\theta}_{h}\right) .
\end{aligned}
$$


The second term is treated as in (48), with $\partial_{t} \boldsymbol{\theta}_{h}$ in the place of $\Pi_{h}^{k} \mathbf{v}_{p}$. Therefore, after using an inverse inequality, we get

$$
\begin{aligned}
T_{2} \leq & C h^{-1}\left\|\mathbf{u}-\mathbf{u}_{h}\right\|_{0, \Omega}\left(\|\mathbf{u}\|_{0, \infty, \Omega}+\left\|\mathbf{u}_{h}\right\|_{0, \infty, \Omega}\right)\left\|\partial_{t} \boldsymbol{\theta}_{h}\right\|_{0, \Omega} \\
& +C h^{-\frac{1}{2}}\left\|\mathbf{u}_{h}\right\|_{0, \infty, \Omega}\left\|\left(\mathbf{u}-\mathbf{u}_{h}, p-p_{h}\right)\right\|_{\mathbf{u}_{h}}\left\|\partial_{t} \boldsymbol{\theta}_{h}\right\|_{0, \Omega} .
\end{aligned}
$$

Finally, using Cauchy-Schwarz and an inverse inequality

$$
\begin{aligned}
T_{3} & \leq j_{\mathbf{u}_{h}}\left(\mathbf{u}_{h}, \mathbf{u}_{h}\right)^{\frac{1}{2}} j_{\mathbf{u}_{h}}\left(\partial_{t} \boldsymbol{\theta}_{h}, \partial_{t} \boldsymbol{\theta}_{h}\right)^{\frac{1}{2}} \\
& \leq C\left\|\left(\mathbf{u}-\mathbf{u}_{h}, p-p_{h}\right)\right\|_{\mathbf{u}_{h}}\left\|\mathbf{u}_{h}\right\|_{0, \infty, \Omega} h^{-\frac{1}{2}}\left\|\partial_{t} \boldsymbol{\theta}_{h}\right\|_{0, \Omega} \\
& \leq C\left\|\mathbf{u}_{h}\right\|_{0, \infty, \Omega}^{2} \frac{h^{-1}}{2 \epsilon}\left\|\left(\mathbf{u}-\mathbf{u}_{h}, p-p_{h}\right)\right\|_{\mathbf{u}_{h}}^{2}+C \frac{\epsilon}{2}\left\|\partial_{t} \boldsymbol{\theta}_{h}\right\|_{0, \Omega}^{2} .
\end{aligned}
$$

Integrating over $(0, T)$, using coercivity (Lemma 8$)$ and by combining estimates (59)-(60) with Theorem 2 , we obtain (for $\epsilon>0$ sufficiently small)

$$
\begin{aligned}
\int_{0}^{T}\left\|\partial_{t} \boldsymbol{\theta}_{h}\right\|_{0, \Omega}^{2} \mathrm{~d} t \leq & C\left\|\left(\boldsymbol{\theta}_{h}(0), y_{h}(0)\right)\right\|_{\mathbf{0}}^{2}+C(\mathbf{u}, p, T) h^{2 \min \left\{r_{\mathbf{u}}, r_{p}\right\}-3} \\
& +\int_{0}^{T} b_{h}\left(p, \partial_{t} \boldsymbol{\theta}_{h}\right) \mathrm{d} t .
\end{aligned}
$$

First of all, we note that after partial integration first in space and then in time, we may write (for the last term on the right hand side),

$$
\begin{aligned}
\int_{0}^{T} b_{h}\left(p, \partial_{t} \boldsymbol{\theta}_{h}\right) \mathrm{d} t & =\int_{0}^{T}\left(\nabla p, \partial_{t} \boldsymbol{\theta}_{h}\right) \mathrm{d} t \\
= & -\int_{0}^{T}\left(\partial_{t} \nabla p, \boldsymbol{\theta}_{h}\right) \mathrm{d} t+\left(\nabla p(T), \boldsymbol{\theta}_{h}(T)\right) \\
& -\left(\nabla p(0), \boldsymbol{\theta}_{h}(0)\right)
\end{aligned}
$$

By applying a Cauchy-Schwarz inequality and the Sobolev embedding

$$
\|\nabla p\|_{L^{\infty}\left(0, T ; L^{2}(\Omega)\right)} \leq C\|\nabla p\|_{H^{1}\left(0, T ; L^{2}(\Omega)\right)},
$$

in combination with Theorem 2 we have

$$
\int_{0}^{T} b_{h}\left(p, \partial_{t} \boldsymbol{\theta}_{h}\right) \mathrm{d} t \leq C\|p\|_{H^{1}\left(0, T ; H^{1}(\Omega)\right)} h^{\min \left\{r_{\mathbf{u}}, r_{p}\right\}-\frac{1}{2}} .
$$


Clearly, we have a triple norm contribution from the unknown initial discrete error in the pressure $y_{h}(0)$ in the right hand side of (61). Indeed, the term we need to control is

$$
\begin{aligned}
j\left(y_{h}(0), y_{h}(0)\right)= & j\left(R_{h}^{k} \mathbf{u}_{0}, R_{h}^{k} \mathbf{u}_{0}-p_{h}(0)\right) \\
& -j\left(p_{h}(0), R_{h}^{k} \mathbf{u}_{0}-p_{h}(0)\right) .
\end{aligned}
$$

Using the discrete incompressibility equations for $\mathbf{u}_{h}(0)$ and $P_{h}^{k} \mathbf{u}_{0}$ (and since $\nabla \cdot \mathbf{u}_{0}=$ 0 ) we have

$$
\begin{aligned}
b_{h}\left(q_{h}, \mathbf{u}_{h}(0)\right) & =j\left(p_{h}(0), q_{h}\right), \\
b_{h}\left(q_{h}, P_{h}^{k} \mathbf{u}_{0}\right) & =j\left(R_{h}^{k} \mathbf{u}_{0}, q_{h}\right) .
\end{aligned}
$$

Thus, taking $q_{h}=R_{h}^{k} \mathbf{u}_{0}-p_{h}(0)$ and since $\mathbf{u}_{h}(0)=P_{h}^{k} \mathbf{u}_{0}$, from (62) we have

$$
\begin{aligned}
0 & =b_{h}\left(R_{h}^{k} \mathbf{u}_{0}-p_{h}(0), P_{h}^{k} \mathbf{u}_{0}-\mathbf{u}_{h}(0)\right) \\
& =j\left(R_{h}^{k} \mathbf{u}_{0}, R_{h}^{k} \mathbf{u}_{0}-p_{h}(0)\right)-j\left(p_{h}(0), R_{h}^{k} \mathbf{u}_{0}-p_{h}(0)\right) \\
& =j\left(y_{h}(0), y_{h}(0)\right) .
\end{aligned}
$$

Hence we conclude that $j\left(y_{h}(0), y_{h}(0)\right)=0$ and the theorem follows.

\section{Conclusion}

We have derived a priori error estimates for finite element approximations of the incompressible Navier-Stokes equations that are independent of the local Reynolds number and hence valid also for the incompressible Euler equations. The estimates are similar to those obtained in [23] in the case of piecewise linear elements and quasioptimal for the velocities, with the loss of $h^{\frac{1}{2}}$ with respect to approximation typical for stabilized methods.

For polynomial orders $k \geq 2$ the estimates for the time derivative of the velocity of Theorem 3 is suboptimal in case the pressure is very regular due to the nonconsistency of the projection (34). The estimate can be improved if the analysis is performed in a time weighted norm [25] or assuming "sufficient" regularity of the pressure, typically $p \in C^{1}\left(0, T ; H^{k+1}(\Omega)\right)$ (see, for instance, [18]). It is questionable if this stronger hypothesis can be justified (see the discussion in [25]). Convergence may also be proven assuming less regularity on the pressure, however if dependence on the viscosity is to be avoided it seems difficult to get away with less than $p \in H^{1}\left(0, T ; L^{2}(\Omega)\right) \cap L^{2}\left(0, T ; H^{1}(\Omega)\right)$. It is our hope that the present analysis sheds some light on the question of how to construct reliable numerical methods for large eddy simulation, where the local Reynolds number must always be assumed to be high. The fully discretized case and numerical examples will be addressed in a forthcoming work. 
Acknowledgments The authors wish to thank an anonymous reviewer for the careful reading of the manuscript. The first author acknowledges support by INRIA during his stay at Rocquencourt, in January 2005, as a visiting professor. The second author was partially supported by the Research Training Network "Mathematical Modelling of the Cardiovascular System (HaeMOdel)", contract HPRN-CT-2002-00270 of the European Community.

\section{References}

1. Becker, R., Braack, M.: A finite element pressure gradient stabilization for the Stokes equations based on local projections. Calcolo 38(4), 173-199 (2001)

2. Becker, R., Braack, M.: A two-level stabilization scheme for the Navier-Stokes equations. In: Numerical mathematics and advanced applications, pages 123-130. Springer, Berlin (2004)

3. Bertoluzza, S.: The discrete commutator property of approximation spaces. C. R. Acad. Sci. Paris Sér. I Math. 329(12), 1097-1102 (1999)

4. Boman, M.: Estimates for the $L_{2}$-projection onto continuous finite element spaces in a weighted $L_{p}$-norm. BIT 46(2), 249-260 (2006)

5. Braack, M., Burman, E.: Local projection stabilization for the Oseen problem and its interpretation as a variational multiscale method. SIAM J. Numer. Anal. (2005)

6. Bramble, J.H., Pasciak, J.E., Steinbach, O.: On the stability of the $L^{2}$ projection in $H^{1}(\Omega)$. Math. Comput. (Electronic) 71(237), 147-156 (2002)

7. Brezzi, F., Fortin, M.: A minimal stabilisation procedure for mixed finite element methods. Numer. Math. 89(3), 457-491 (2001)

8. Burman, E.: A unified analysis for conforming and nonconforming stabilized finite element methods using interior penalty. SIAM J. Numer. Anal. 43(5), 2012-2033 (2005)

9. Burman, E., Fernández, M.A.: A finite element method with edge oriented stabilization for the timedependent Navier-Stokes equations: space discretization and convergence. Technical Report RR-5630, INRIA (2005)

10. Burman, E., Fernández, M.A., Hansbo, P.: Continuous interior penalty finite element method for Oseen's equations. SIAM J. Numer. Anal. 44(3), 1248-1274 (2006)

11. Burman, E., Hansbo, P.: Edge stabilization for the generalized Stokes problem: a continuous interior penalty method. Comput. Methods Appl. Mech. Engrg. 195(19-22), 2393-2410 (2006)

12. Burman, E., Hansbo, P.: Edge stabilization for Galerkin approximations of convection-diffusion-reaction problems. Comput. Methods Appl. Mech. Engrg. 193(15-16), 1437-1453 (2004)

13. Clément, P.: Approximation by finite element functions using local regularization. Rev. Française Automat. Informat. Recherche Opérationnelle Sér. Rouge Anal. Numér. 9(R-2), 77-84 (1975)

14. Codina, R.: Stabilized finite element approximation of transient incompressible flows using orthogonal subscales. Comput. Methods Appl. Mech. Engrg. 191(39-40), 4295-4321 (2002)

15. Codina, R., Blasco, J.: Analysis of a pressure-stabilized finite element approximation of the stationary Navier-Stokes equations. Numer. Math. 87(1), 59-81 (2000)

16. Crouzeix, M., Thomée, V.: The stability in $L_{p}$ and $W_{p}^{1}$ of the $L_{2}$-projection onto finite element function spaces. Math. Comput. 48(178), 521-532 (1987)

17. Douglas J. Jr., Dupont, T.: Interior penalty procedures for elliptic and parabolic Galerkin methods, volume 58 of Lecture Notes in Physics. Springer, Berlin (1976)

18. Ern, A., Guermond, J.-L.: Theory and practice of finite elements, volume 159 of applied mathematical sciences. Springer, New York (2004)

19. Freund, J., Stenberg, R.: On weakly imposed boundary conditions for second order problems. In: Morandi Cecchi, M. et al. (ed.) Proceedings of the ninth international conference finite elements in fluids, pp. 327-336, Venice (1995)

20. Girault, V., Raviart, P.-A.: Finite element methods for Navier-Stokes equations, vol. 5 of Springer series in computational mathematics. Springer, Berlin (1986)

21. Guermond, J.-L.: Stabilization of Galerkin approximations of transport equations by subgrid modeling. M2AN Math. Model. Numer. Anal. 33(6), 1293-1316 (1999)

22. Guermond, J.-L., Prudhomme, S.: Mathematical analysis of a spectral hyperviscosity LES model for the simulation of turbulent flows. M2AN Math. Model. Numer. Anal. 37(6), 893-908 (2003)

23. Hansbo, P., Szepessy, A.: A velocity-pressure streamline diffusion finite element method for the incompressible Navier-Stokes equations. Comput. Methods Appl. Mech. Engrg. 84(2), 175-192 (1990) 
24. He, Y., Lin, Y., Sun, W.: Stabilized finite element method for the non-stationary Navier-Stokes problem. Discrete Contin. Dyn. Syst. Ser. (Electronic) B 6(1), 41-68 (2006)

25. Heywood, J.G., Rannacher, R.: Finite element approximation of the nonstationary Navier-Stokes problem. I. Regularity of solutions and second-order error estimates for spatial discretization. SIAM J. Numer. Anal. 19(2), 275-311 (1982)

26. Heywood, J.G., Rannacher, R.: Finite element approximation of the nonstationary Navier-Stokes problem. II. Stability of solutions and error estimates uniform in time. SIAM J. Numer. Anal. 23(4), 750777 (1986)

27. Heywood, J.G., Rannacher, R.: Finite element approximation of the nonstationary Navier-Stokes problem. III. Smoothing property and higher order error estimates for spatial discretization. SIAM. J. Numer. Anal. 25(3), 489-512 (1988)

28. Hoppe, R.H.W., Wohlmuth, B.: Element-oriented and edge-oriented local error estimators for nonconforming finite element methods. RAIRO Modél. Math. Anal. Numér. 30(2), 237-263 (1996)

29. Johnson, C., Szepessy, A., Hansbo, P.: On the convergence of shock-capturing streamline diffusion finite element methods for hyperbolic conservation laws. Math. Comp. 54(189), 107-129 (1990)

30. Johnson, C., Saranen, J.: Streamline diffusion methods for the incompressible Euler and Navier-Stokes equations. Math. Comp. 47(175), 1-18 (1986)

31. Oswald, P.: On a BPX-preconditioner for P1 elements. Computing 51(2), 125-133 (1993)

32. Scott, L.R., Zhang, S.: Finite element interpolation of nonsmooth functions satisfying boundary conditions. Math. Comp. 54(190), 483-493 (1990)

33. Temam, R.: Une méthode d'approximation de la solution des équations de Navier-Stokes. Bull. Soc. Math. France 96, 115-152 (1968)

34. Thomée, V.: Galerkin finite element methods for parabolic problems, volume 25 of Springer series in computational mathematics, 2nd edn. Springer, Berlin (2006)

35. Tobiska, L., Verfürth, R.: Analysis of a streamline diffusion finite element method for the Stokes and Navier-Stokes equations. SIAM J. Numer. Anal. 33(1), 107-127 (1996) 